\title{
Application of the Least Squares Solutions in Image Deblurring
}

\author{
Predrag S. Stanimirović, ${ }^{1}$ Igor Stojanovic,, ${ }^{2}$ Vasilios N. Katsikis, ${ }^{3}$ \\ Dimitrios Pappas, ${ }^{4}$ and Zoran Zdravev ${ }^{2}$ \\ ${ }^{1}$ Faculty of Sciences and Mathematics, University of Niš, Višegradska 33, 18000 Niš, Serbia \\ ${ }^{2}$ Faculty of Computer Science, Goce Delčev University, Goce Delčev 89, 2000 Štip, Macedonia \\ ${ }^{3}$ Department of Economics, Division of Mathematics and Informatics, National and Kapodistrian University of Athens, \\ Sofokleous 1 Street, 10559 Athens, Greece \\ ${ }^{4}$ Department of Statistics, Athens University of Economics and Business, 76 Patission Street, 10434 Athens, Greece
}

Correspondence should be addressed to Predrag S. Stanimirović; pecko@pmf.ni.ac.rs

Received 2 September 2014; Revised 20 January 2015; Accepted 28 January 2015

Academic Editor: Joao B. R. Do Val

Copyright (C) 2015 Predrag S. Stanimirović et al. This is an open access article distributed under the Creative Commons Attribution License, which permits unrestricted use, distribution, and reproduction in any medium, provided the original work is properly cited.

\begin{abstract}
A new method for the reconstruction of blurred digital images damaged by separable motion blur is established. The main attribute of the method is based on multiple applications of the least squares solutions of certain matrix equations which define the separable motion blur in conjunction with known image deconvolution techniques. The key feature of the proposed algorithms is reflected in the fact that they can be used only in symbiosis with other image restoration algorithms.
\end{abstract}

\section{Introduction}

In practice, the recorded image unavoidably represents a degraded version of the original scene because of inevitable imperfections in the imaging and capturing process. Medical images, satellite images, astronomical images, or poor-quality family portraits are often blurred. A wide range of different degradations need to be taken into account, covering, for instance, noise, blur, illumination, and color imperfections, and geometrical degradations. The elimination of these imperfections is crucial in various tasks of image processing and image analysis. Image restoration methods are used for reconstructing the original image from a degraded model. The problem of image restoration has been studied in many articles and books [1-7].

The application of the least squares solutions in image processing (and in image restoration particularly) is not frequently investigated so far. An application of the least square techniques in image processing is presented in [8]. Removal of blur in images based on the least squares solutions is investigated in $[9,10]$. Particularly, an application of the least squares solution of minimal norm in image deblurring is investigated in [11-13]. Our main intention in this paper is further investigation and extension of the algorithms introduced in $[9,10]$ that allows us to remove a linear or separable motion blur from images. The algorithms presented in these papers are based on the least squares solution of a matrix equation which represents the mathematical model of the linear or separable motion blur. The least squares solution, denoted by $E_{1}$, includes the Moore-Penrose inverse of the blurring matrix as well as an arbitrary matrix $Y$. The particular least squares solution, based on the Moore-Penrose inverse, was investigated in [11-13].

The main goal of this paper is the development of an algorithm that allows us to remove a motion blur from images by means of the consecutive applications of the least squares solutions of a matrix equation which models the separable two-dimensional blurring process. The least squares solutions are matrix expressions that include the Moore-Penrose inverse of the blurring matrix as well as an appropriately chosen arbitrary matrix. The matrix transformation defined in this way is idempotent (see $[9,10])$. This difficulty forces us to find the way to manage consecutive applications of the least squares solutions from $[9,10]$. Significant improvements in ISNR and PSNR values are attained applying such an approach with respect to the classical approach based on 
the Moore-Penrose solution of certain matrix equations, which is investigated in $[11,12]$, as well as with respect to a single application of the least squares solutions, used in $[9,10]$.

The paper is organized as follows. Motivation and description of the method are presented in the second section. The main algorithm which enables the iterative application of the least squares solution $E_{1}$ on images damaged by a separable motion blur is also presented in the second section, as well as the application of the method on blurred and noisy images. Results generated by performed numerical experiments are investigated in Section 3.

\section{Motivation and Description of the Method}

The process of the separable blurring assumes that the blurring of the columns in the image is independent of the blurring of the rows. The separable blurring is modeled by two blurring matrices, $H_{c}$ and $H_{r}$, both of the general form

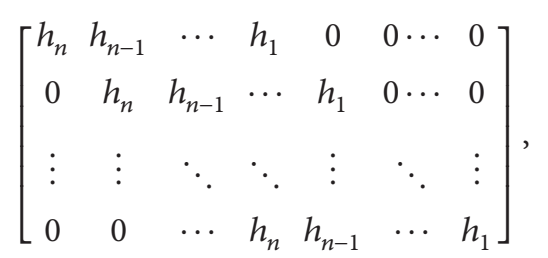

where $h_{i}, i=1, \ldots, n$, are real numbers and the positive integer $n$ indicates the length of linear motion blur in pixels. The relation between the original image $F$ and blurred image $G$ is expressed by the following matrix equation, considered in [10]:

$$
\begin{aligned}
G=H_{c} F H_{r}^{T}, \\
G \in \mathbb{R}^{m_{1} \times m_{2}}, \quad H_{c} \in \mathbb{R}^{m_{1} \times r}, \\
F \in \mathbb{R}^{r \times s}, \quad H_{r} \in \mathbb{R}^{m_{2} \times s} .
\end{aligned}
$$

In (2), it is assumed that $s=m_{2}+n_{1}-1, r=m_{1}+n_{2}-1$, where $n_{1}$ (resp., $n_{2}$ ) is the length of the horizontal (resp., vertical) blurring in pixels.

Our approach provides a new method for restoration of a blurred image which is based on multiple applications of the least squares solutions of the matrix equations (2) in symbiosis with other well-known image deblurring techniques. In general, the proposed algorithm is aimed at solving the matrix equation (2).

The least squares solution of (2) has the general form (used in [10])

$$
\begin{aligned}
E_{1}(Y) & =\widetilde{F}=H_{c}^{\dagger} G\left(H_{r}^{T}\right)^{\dagger}+Y-H_{c}^{\dagger} H_{c} Y H_{r}^{T}\left(H_{r}^{T}\right)^{\dagger} \\
& =\widehat{F}+Y-H_{c}^{\dagger} H_{c} Y H_{r}^{\dagger} H_{r},
\end{aligned}
$$

where $Y$ is an arbitrary matrix of appropriate dimensions.

The transformation $E_{1}(Y)$ can be used as a deconvolution of a blurred image $Y$. The blurred image $Y \in \mathbb{R}^{r \times s}$, used in (3), can be determined in different ways. There are no specific conditions for that; any random matrix $Y$ can be transformed into $E_{1}(Y)$. Continuing investigations from $[9,10]$, appropriate choices for $Y$ are chosen as the results of particular image deblurring processes; that is, $Y$ is an arbitrary restoration of the degraded image. In that case, $E_{1}(Y)$ is an attempt to derive further improvements in the restoration of $Y$.

In the case $Y=O$, where $O$ is the zero matrix of appropriate dimensions, $E_{1}(Y)$ produces the next approximation $\widehat{F}$ of the original image $F$ :

$$
E_{1}(O)=\widehat{F}=H_{c}^{\dagger} G\left(H_{r}^{T}\right)^{\dagger}
$$

The approach which assumes the condition $Y=O$ in (3) exploits the Moore-Penrose solution of the matrix equation (2), that is, the least squares solution of minimal norm. About the least squares and minimal norm properties of the Moore-Penrose solution, see main references [14, 15]. But the minimal norm attribute associated with the MoorePenrose solution may be, in most of cases, only the redundant property. Indeed, our experience from $[9,10]$ confirms that only when the matrix $Y$ is selected to be "far" from the original image, the improvement of $Y$ is still worse with respect to the Moore-Penrose reconstruction (corresponding to the case $Y=O$ ). Some of the examples that confirm this expectation are studied in $[9,10]$. We follow the main goal of the papers $[9,10]$; that is, we will determine $Y$ in such a way that the approximation $\widetilde{F}$ produces better values for ISNR and PSNR with respect to the solution $\widehat{F}$ which is used in $[11,12]$.

Except the election $Y=O$, the results generated by applying the Wiener filter (WF) and the constrained leastsquares (CLS) filter are used as two appropriate choices of the matrix $Y$ in $[9,10]$. A description of the WF and CLS filters can be found in [2]. A more advanced approach for the selection of the matrix $Y$ is based on the moment based methods. The Haar basis and the Fourier basis mentioned above are usually referred to as the most popular moment based methods. This approach is considered in [10]. For more details on the Fourier and the Haar basis, see $[9,12]$. An algorithm for image deconvolution from the geometric moments of an image which is degraded by a circular or elliptical Gaussian point-spread function is considered in [16]. For additional information on the moment based image reconstruction methods, the reader is referred to [16-20]. A short preview of main image restoration methods, used for obtaining possible reconstructions $Y$, is presented in [10]. A detailed description of these methods can be found in [13]. A recent survey book presenting all the modern image reconstruction methods is given in [21].

Our improvement of the methods defined in $[9,10]$ arises from our intention to apply the operator $E_{1}$ repeatedly. But the authors in [9] showed that the operator $E_{1}(Y)$ is idempotent, which implies $E_{1}\left(E_{1}(Y)\right)=E_{1}(Y)$. This property of the operator $E_{1}$ makes its application on $E_{1}(Y)$ redundant. In the present paper, we find a possibility for multiple applications of the operator $E_{1}$. The main idea is to use $E_{1}\left(T_{k}\left(E_{1}(Y)\right)\right)$ instead of $E_{1}\left(E_{1}(Y)\right)$, where $T_{k}$ denotes the application of a previously defined image deconvolution algorithm in the $k$ th iteration. 
Let us denote by $\mathfrak{F}_{\kappa}$ the reconstructed image after $\kappa$ steps. The following approximations $Y_{\kappa}$ for further restorations are considered:

(a) $Y_{\kappa}=B_{k, l}\left(\mathfrak{F}_{\kappa}\right)$ is derived applying the Haar based reconstructed image for selected $k, l$;

(b) $Y_{\kappa}=$ Fourier $_{k, l}\left(\mathfrak{F}_{\kappa}\right)$ is derived applying the Fourier based reconstructed image for selected $k, l$;

(c) $Y_{\kappa}=\operatorname{CLS}\left(\mathfrak{F}_{\kappa}\right)$ is derived applying the constrained least-squares filter;

(d) $Y_{\kappa}=\mathrm{WF}\left(\mathfrak{F}_{\kappa}\right)$, derived applying the Wiener filter;

(e) $Y_{\kappa}=\operatorname{LR}\left(\mathfrak{F}_{\kappa}\right)$, derived applying the Lucy-Richardson algorithm.

On the other hand, an improper and unpredictable behavior of $E_{1}$ is observed in $[9,10]$. Namely, to some extent, a better improvement $Y$ implies better restoration $E_{1}(Y)$, which is confirmed by the inequality $\operatorname{ISNR}\left(E_{1}(Y)\right)>\operatorname{ISNR}(Y)$. But, after the occurrence of a limit, which is not known in advance, the opposite situation $\left(\operatorname{ISNR}\left(E_{1}(Y)\right)<\operatorname{ISNR}(Y)\right)$ is observed.

One of the possible ways to achieve multiple applications of the operator $E_{1}$ is its alternating application with another image restoration method, as it is described in Algorithm 1.

Algorithm 1 (iterative application of the operator $E_{1}$ ). Consider the following.

Require $Y_{0}=G$, where $G$ denotes a blurred image.

(1) Initial step is

$\mathfrak{F}_{1}=E_{1}\left(Y_{0}\right)=\widetilde{F}_{0}=H_{c}^{\dagger} G\left(H_{r}^{T}\right)^{\dagger}+Y_{0}-H_{c}^{\dagger} H_{c} Y_{0} H_{r}^{\dagger} H_{r}$.

(2) Set the number of the iterative steps to initial value $\kappa=1$.

(3) Compute

$$
\begin{gathered}
Y_{\kappa}=T_{\kappa}\left(\mathfrak{\mho}_{\kappa}\right) \\
\mathfrak{\mho}_{\kappa+1}=E_{1}\left(Y_{\kappa}\right)=H_{c}^{\dagger} G\left(H_{r}^{T}\right)^{\dagger}+Y_{\kappa}-H_{c}^{\dagger} H_{c} Y_{\kappa} H_{r}^{\dagger} H_{r}, \\
\kappa \geq 1,
\end{gathered}
$$

where $T_{\kappa} \in\left\{\right.$ Fourier $\left._{k, l}, B_{k, l}, \mathrm{WF}, \mathrm{LR}, \mathrm{TSVD}\right\}$.

(4) If a selected stopping criterion is fulfilled set $\kappa=\kappa+1$ and go to Step (3).

(5) If the stopping criterion is fulfilled return the output $\mathfrak{F}_{\kappa}$.

Remark 2. Iterations (6) should provide two improvements in the reconstruction, in each iteration, as follows:

(i) the first improvement is $Y_{\kappa}=T_{\kappa}\left(\mathfrak{F}_{\kappa}\right)$, which gives a restoration $Y_{\kappa}$ of the previous iteration $\mathfrak{F}_{\kappa}$ by means of an image restoration method $T_{\kappa}$;

(ii) the second improvement arises from $\mathfrak{F}_{\kappa+1}=E_{1}\left(Y_{\kappa}\right)$, which gives further reconstruction $\mathfrak{F}_{\kappa+1}$ of $Y_{\kappa}$ by means of the least squares solution $E_{1}$.
Remark 3. The choice of the stopping criterion in Step (4) of Algorithm 1 is, at this moment, an undeterminable problem.

(i) One possible choice is to stop the cycle when the inequality $\operatorname{ISNR}\left(\mathfrak{F}_{\kappa+1}\right)<\operatorname{ISNR}\left(\mathfrak{F}_{\kappa}\right)$ is satisfied.

(ii) But this choice may cause blocking of (possible) improvements in further steps.

For this purpose, it seems that the terminating criterion defined by an in advance defined number of iterative steps is a better choice. In our numerical experiments, we will use this stopping criterion.

(iii) Also, it is reasonable to stop the cycle in Step (4) when the inequality $\operatorname{ISNR}\left(\mathfrak{F}_{\kappa+1}\right)<\operatorname{ISNR}\left(\mathfrak{F}_{\kappa}\right)$ is satisfied several times consecutively.

Remark 4. Essentially, iterations in (6) are based on the least squares solution of the dynamical matrix equation

$$
\mathfrak{\mho}_{\kappa}=H_{c} \mathfrak{\mho}_{\kappa+1} H_{r}^{T}
$$

The model (7) essentially means that the iteration $\mathfrak{F}_{\mathcal{K}}$ is considered as a "blurred image" of the next (unknown) iteration $\mathfrak{F}_{\kappa+1}$. After resolving (7) with respect to the unknown matrix $\mathfrak{F}_{k+1}$, we derive the next iteration $\mathfrak{\mho}_{k+1}$ in terms of the current iteration $\mathfrak{F}_{\kappa}$ and the pseudoinverses of the blurring matrices $H_{c}$ and $H_{r}$.

Remark 5. The authors of the papers $[9,10]$ have computed $Y=T(G)$, where $T$ is an image restoration method, and simply compared $\operatorname{ISNR}(Y)$ with $\operatorname{ISNR}\left(E_{1}(Y)\right)$. The main advantage of the proposed Algorithm 1 is that it makes repetitive use of the operator $E_{1}$ in symbiosis with selected deblurring methods on the blurred image and its reconstructions. Since the operator $E_{1}$ is idempotent, Algorithm 1 defines an approach to improve the results obtained in $[9,10]$ by the multiple application of the (deblurring) transformation $E_{1}$.

2.1. Repetitive Least Squares Image Deblurring and Denoising. Noise is unavoidable in most of applications, so that a real observation is thus often modeled by the following mathematical model:

$$
G_{N}=H_{c}(F+N) H_{r}^{T}=H_{c} F_{N} H^{T},
$$

where $N$ is additive noise and $G_{N}$ is the blurred noisy image.

Algorithm 6 can be adopted to restore the original image from a blurred and noisy image, using the least squares solution of the mathematical model (8).

Algorithm 6 (iterative application of the operator $E_{1}$ on blurred and noisy image). Consider the following.

Require $Y_{0}=G_{N}$, where $G_{N}$ denotes a blurred noisy image.

(1) Initial step is

$$
\widetilde{\mathfrak{F}}_{1}=E_{1}\left(Y_{0}\right)=\widetilde{F}_{0}=H_{c}^{\dagger} G_{N}\left(H_{r}^{T}\right)^{\dagger}+Y_{0}-H_{c}^{\dagger} H_{c} Y_{0} H_{r}^{\dagger} H_{r} .
$$




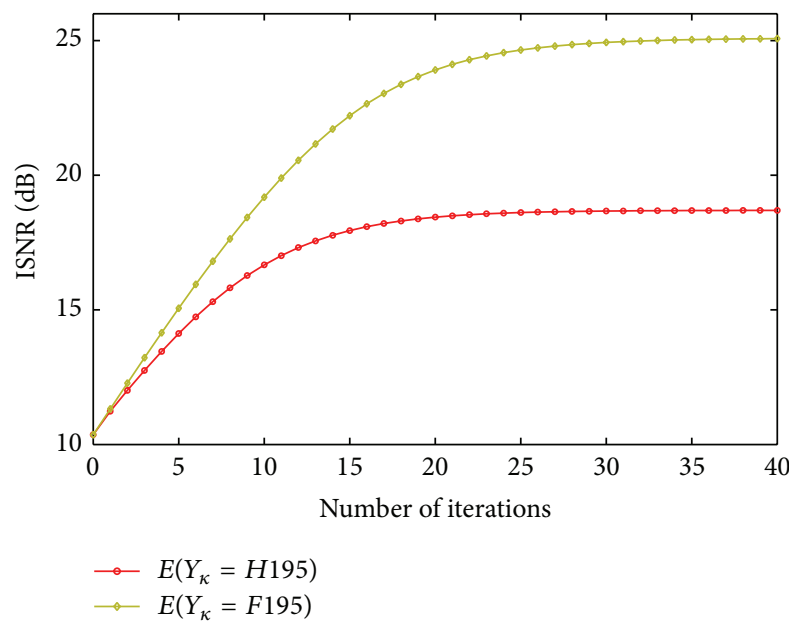

(a)

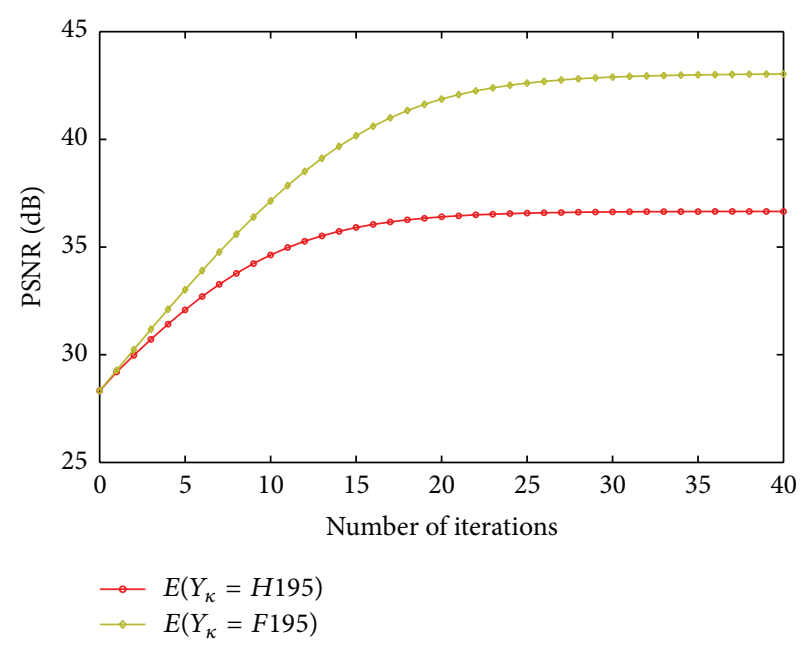

(b)

FIGURE 1: (a) ISNR versus the number of steps of Algorithm 1; (b) PSNR versus the number of steps of Algorithm 1.

(2) Obtain the restored image $\mathfrak{\mho}_{1}$ by applying filtering process on the image $\widetilde{\mathfrak{F}}_{1}$ obtained in Step (1).

(3) Set $\kappa=1$.

(4) Compute

$$
\begin{gathered}
Y_{\kappa}=T_{\kappa}\left(\widetilde{\mho}_{\kappa}\right), \\
\widetilde{\mathfrak{F}}_{\kappa+1}=E_{1}\left(Y_{\kappa}\right)=H_{c}^{\dagger} G_{N}\left(H_{r}^{T}\right)^{\dagger}+Y_{\kappa}-H_{c}^{\dagger} H_{c} Y_{\kappa} H_{r}^{\dagger} H_{r},
\end{gathered}
$$$$
\kappa \geq 1
$$

where $T_{\kappa} \in\left\{\right.$ Fourier $\left._{k, l}, B_{k, l}, \mathrm{WF}, \mathrm{LR}, \mathrm{TSVD}\right\}$.

(5) Obtain the restored image $\mathfrak{F}_{k+1}$ by applying filtering process on the image $\widetilde{\widetilde{F}}_{k+1}$ obtained in Step (4).

(6) If a selected stopping criterion is fulfilled, set $\kappa=\kappa+1$ and return to Step (4).

(7) If the stopping criterion is fulfilled then return the output $\mathfrak{\mho}_{\kappa}$.

\section{Experimental Results}

In this section, we investigate the numerical results generated by applying the two proposed algorithms. The experiments are performed using Matlab programming language on an Intel Core i5 CPU M430 @ 2.27 GHz 64/32-bit system with $4 \mathrm{~GB}$ of RAM memory running on Windows 7 Ultimate Operating System.

\subsection{Application of the Method on Blurred Images}

Example 7. In order to emphasize the importance of the number of iterative steps in Algorithm 1, the number of moments in Figures 1(a) and 1(b) was kept constant with $k=l=195$. The length of blurring is $H_{c}=H_{r}=64$. The reconstructions $Y_{\kappa}$ obtained by the Haar basis (resp., Fourier transform) are denoted by $Y=H_{195,195}=H 195$ (resp., $\left.Y=F_{195,195}=F 195\right)$.

Both the graphs represented in Figures 1(a) and 1(b) show a similar behavior. In fact, the ISNR and PSNR values increase initially and then converge, slowly growing, to a constant value. Also, both ISNR and PSNR values generated by applying $E_{1}$ on Fourier basis are greater (better) with respect to the corresponding values generated by applying $E_{1}$ on the Haar basis.

Also, the graphs in Figures 1(a) and 1(b) show that in advance the predefined number of iterative steps is a suitable termination criterion of Algorithm 1.

Example 8. This example shows the behavior of Algorithm 1 with respect to the length of blurring. Therefore, the number of iterative steps remains constant while the length of blurring in Step (1) increases in the range $[10,100]$. In order to compare generated results, we tested Algorithm 1 on several standard Matlab images: Lena, Barbara, Man, Boat, and Cameraman. The best ISNR (resp., PSNR) values versus the length of the blurring process for Lena image, generated after 40 steps of Algorithm 1, are illustrated in Figure 2(a) (resp., Figure 2(b)). In general, both ISNR and PSNR values stepwise decrease with increasing the length of blurring. Let us mention that the values corresponding to the constrained least-squares (CLS) filter are not included in these graphs, since they are almost identical with the values corresponding to Wiener filter (WF). Notations $E_{1}(Y 40=F 195)$ (resp., $\left.E_{1}(Y 40=H 195)\right)$ in Figure 2 mean the maximal ISNR values generated after 40 iterative steps of Algorithm 1 using $T_{\kappa}=F_{195}$ (resp. $\left.T_{\kappa}=H_{195}\right), \kappa \geq 1$. The same notations in Figure 2(b) denote the corresponding PSNR values.

Figure 2(a) (resp., Figure 2(b)) shows that $E_{1}(Y 40=$ F195) and $E_{1}(Y 40=H 195)$ achieve the greatest ISNR (resp., PSNR) values for the length of blurring satisfying $n>20$. Notation $E_{1}(Y 0)$ denotes the result derived after a single application of the least squares operator $E_{1}$, that is, the restoration given by applying only Step (1) of 


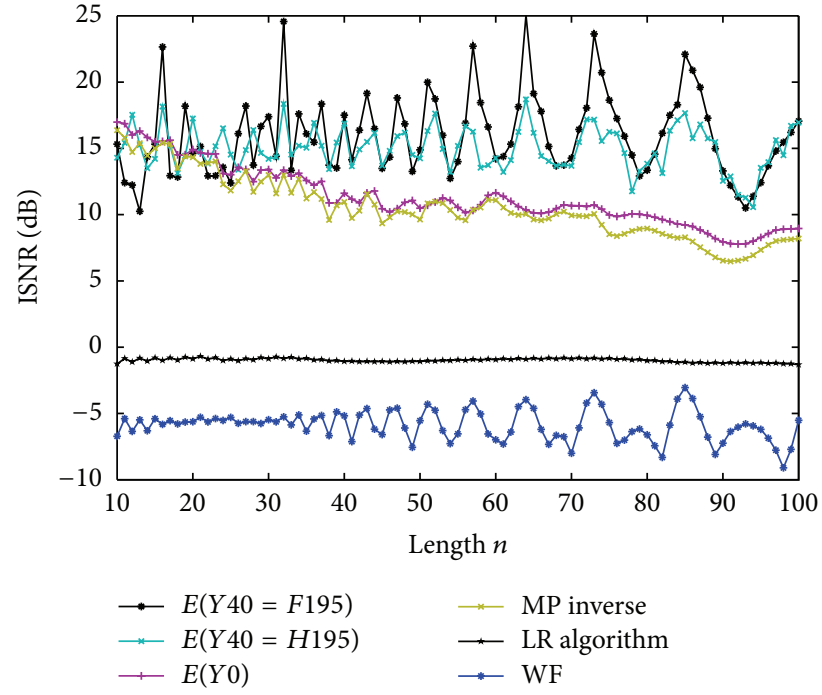

(a)

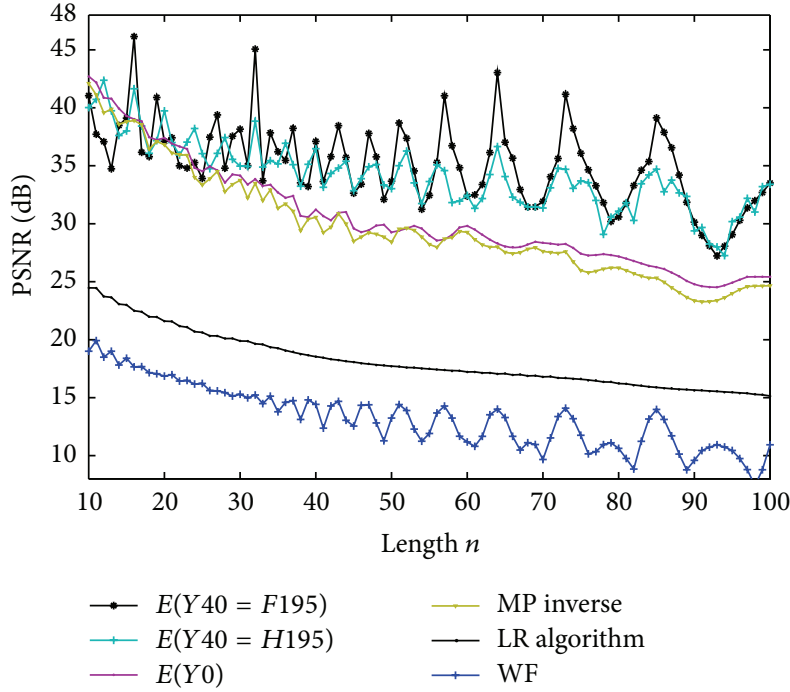

(b)

FIGURE 2: Lena image: (a) maximal ISNR versus length of blurring after 40 steps of Algorithm 1; (b) maximal PSNR versus length of blurring after 40 steps of Algorithm 1.

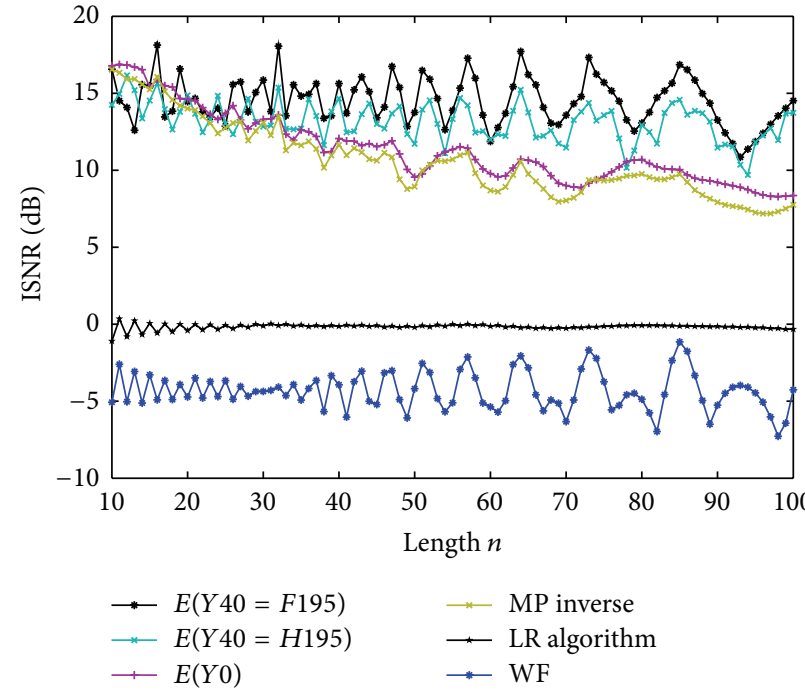

(a)

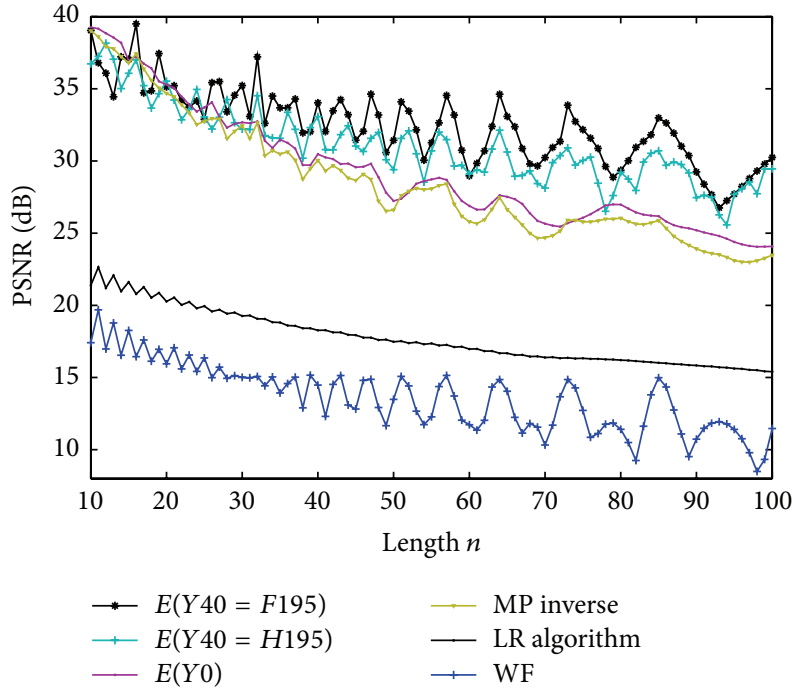

(b)

FIgURE 3: Barbara image: (a) maximal ISNR versus length of blurring after 40 steps of Algorithm 1; (b) maximal PSNR versus length of blurring after 40 steps of Algorithm 1.

Algorithm 1. It can be observed that both ISNR and PSNR values corresponding to $E_{1}(Y 0)$ behave in a similar way as the corresponding restorations derived by applying the MoorePenrose solution (4).

The results of ISNR and PSNR values corresponding to Barbara and Cameraman images are presented in Figures 3 and 4 . The graphs presented in these figures are similar with the graphs presented in Figures 2(a) and 2(b).

Example 9. Figure 5 shows maximal ISNR and the PSNR values for various moments indices $k, l$, from the values $k=$ $l=10$ to $k=l=400$ with the incremental Step (5), keeping the blurring process constant with the length $n=40$ and applying $\kappa=40$ steps of Algorithm 1 .

Generally, an insignificant and stepwise increase of ISNR and PSNR values can be observed. The graphs included in Figure 5 confirm that the improvement of the restoration which is based on increasing the number of moments is insignificant compared to the increase in numerical demands, and, therefore, it is not cost-effective.

Example 10. The tested image Lena is shown in Figure 6(a). The blurred image that has been degraded by a uniform linear motion in the horizontal direction is presented in Figure 6(b). 


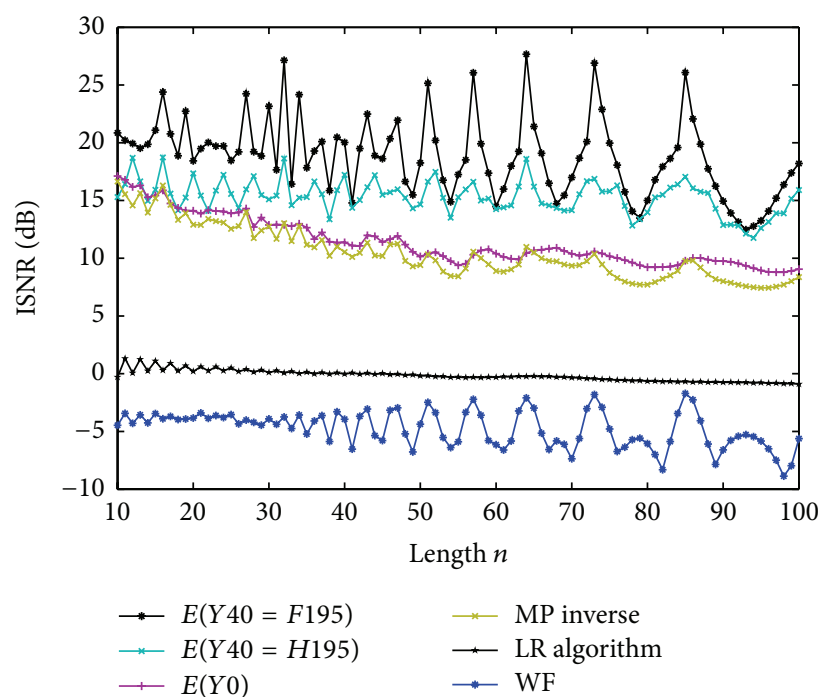

(a)

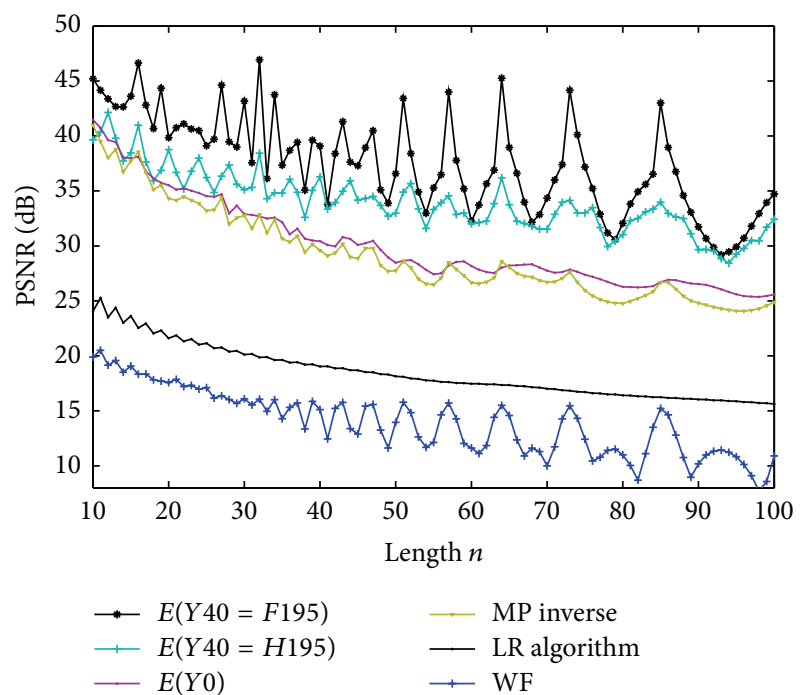

(b)

FIGURE 4: Cameraman image: (a) maximal ISNR versus length of blurring after 40 steps of Algorithm 1; (b) maximal PSNR versus length of blurring after 40 steps of Algorithm 1.

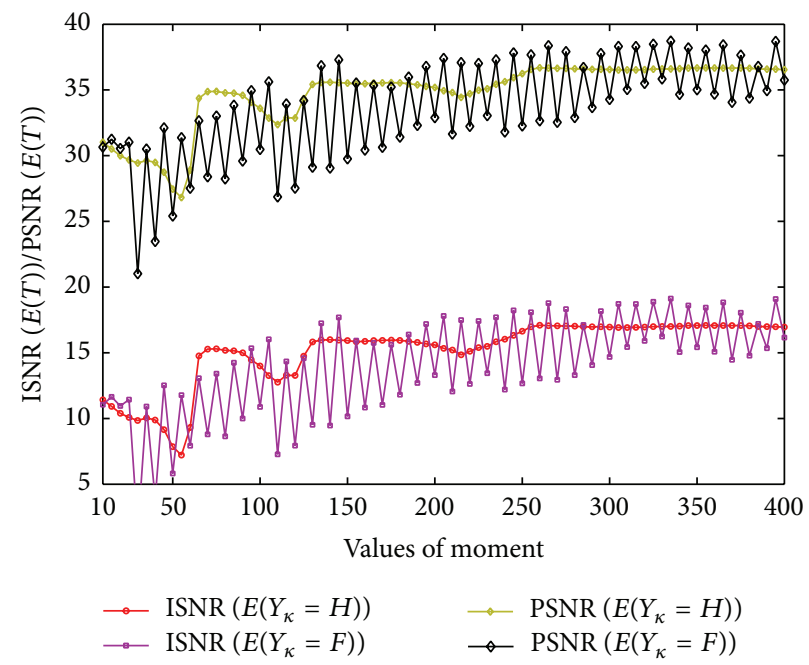

FIGURE 5: Maximal ISNR and PSNR versus the values of moments corresponding to the constant length $l=40$ of blurring.

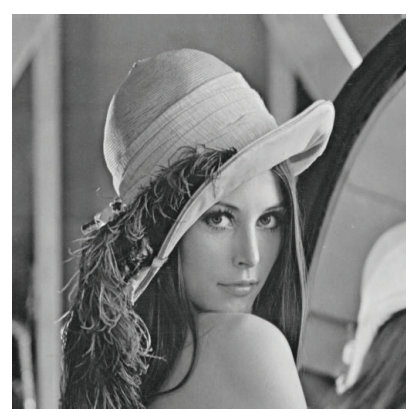

(a)

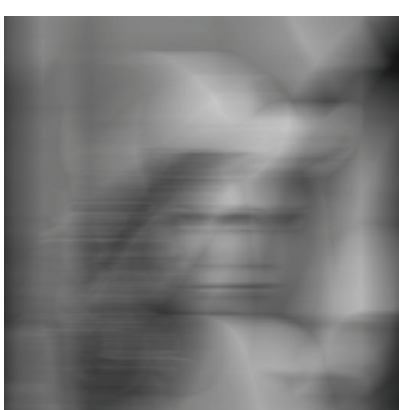

(b)

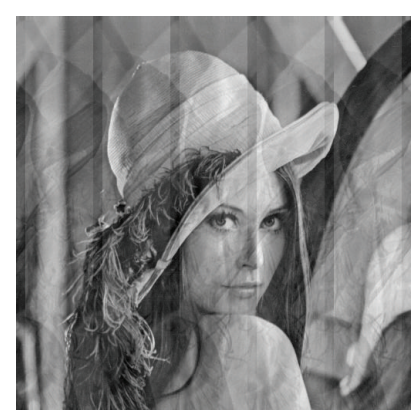

(c)

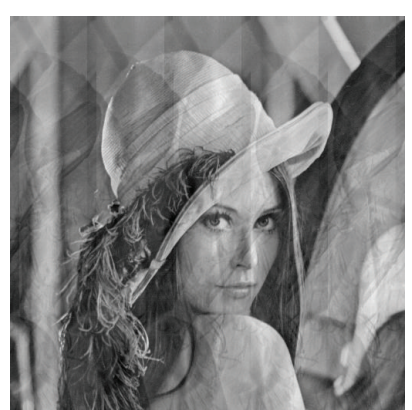

(d)

Figure 6: (a) Image of Lena. (b) Image of Lena blurred by uniform linear motion of length 64. Restoration of Lena in the cases: (c) MoorePenrose; (d) $\kappa=1$. 


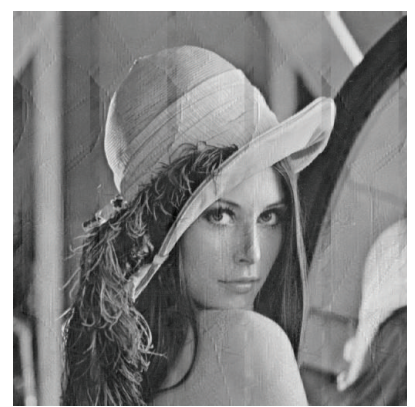

(a)

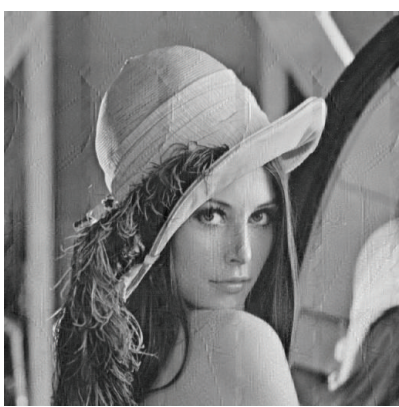

(b)

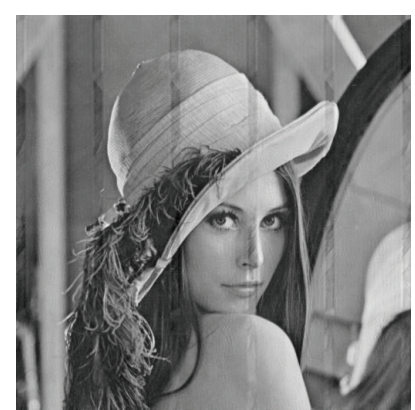

(c)

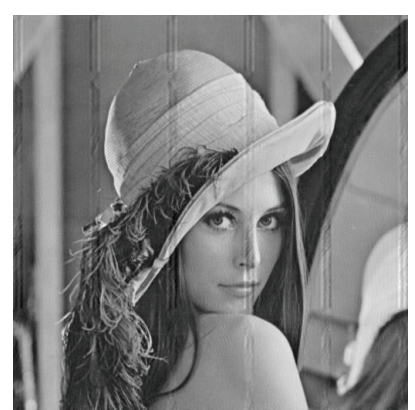

(d)

FIGURE 7: Restoration of Lena with Haar basis for (a) $\kappa=10$ and (b) $\kappa=40$. Restoration of Lena with Fourier basis for (c) $\kappa=10$ and (d) $\kappa=40$.

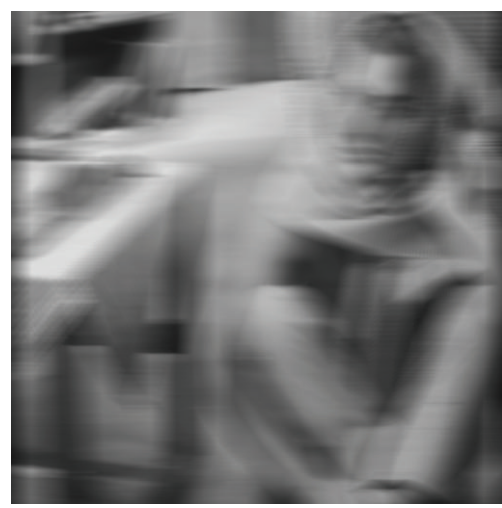

(a)

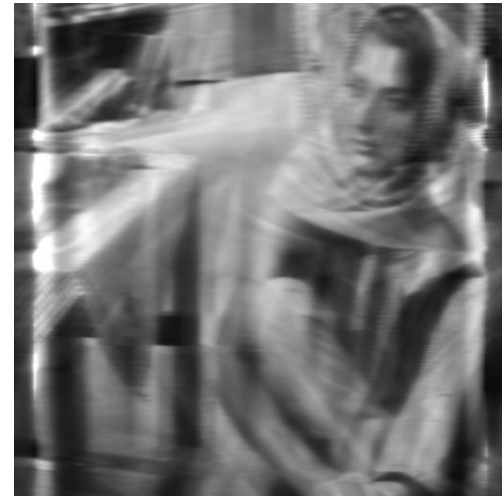

(b)

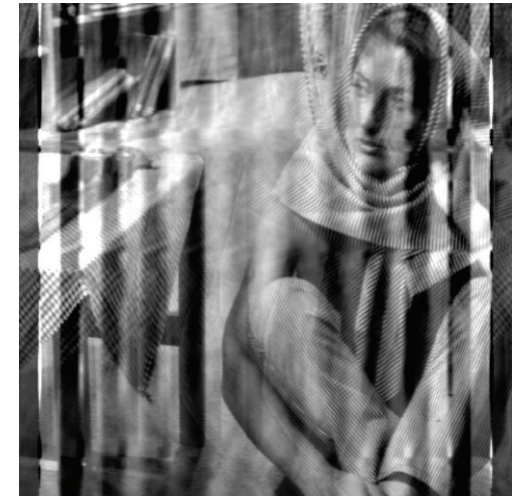

(c)

FIgURE 8: (a) Blurred image by uniform linear motion of length 43. Restoration of Barbara for (b) LR algorithm and (c) WF.

Figure 6(c) illustrates the restoration based on (4) (introduced in $[11,12])$ and Figure 6(d) illustrates the restoration based on a single application of the operator $E_{1}$ (the number of iterations is $\kappa=1)$. Differences in Figures 6(c) and 6(d) are not observable by a human eye. This observation exactly means that a single application of the least squares solution does not produce significantly better results compared with the corresponding results obtained by the Moore-Penrose inverse solution.

Figures $7(a)$ and $7(b)$ present restorations derived applying, respectively, $\kappa=10$ and $\kappa=40$ times the least squares operator $E_{1}$ in conjunction with the Haar basis restoration determined by the basis $k=l=195$. The length of blurring is equal to 64. Figures 7(c) and 7(d) show restorations derived after $\kappa=10$ and $\kappa=40$ applications of the least squares operator $E_{1}$ in conjunction with the Fourier basis restoration with basis $k=l=195$. The length of blurring is 64 .

From Figure 7, one can observe that better restorations are derived applying a greater number of iterations, in both cases (Haar and Fourier basis).

Figures 8 and 9 show the restorations corresponding to the Barbara image. Observation is the same as in the case of Figure 7.

3.2. Application of the Method on Blurred and Noisy Images. The next examples refer to the case when the image is degraded by including image noise which is later followed by a separable motion blur, as it is presented in Section 2.1.

Example 11. The behavior of Algorithm 6 with respect to length of blurring and different types of noise is presented in this example. The changes of the ISNR and PSNR values corresponding to the degraded image of Lena are graphically presented in Figures 10(a) and 10(b). It is possible to use different types of filters, subject to different types of noise. A Gaussian low-pass filter is imposed in this example. Maximal ISNR values generated after $\kappa=40$ steps of Algorithm 6 versus length of blurring for Lena image are presented in Figure 10(a). The "salt and paper" noise of density 0.03 is imposed in addition to the separable motion blur.

Maximal PSNR values versus length of blurring after 40 steps of Algorithm 6 for Lena image are illustrated in Figure 10(b). The Gaussian white noise of mean 0 and variance 0.01 is assumed.

All graphs included in Figure 10(a) (resp., Figure 10(b)) show similar behavior with respect to the graphs included in Figure 2(a) (resp., Figure 2(b)). The graphs corresponding to $E_{1}(Y 40=F 195)$ and $E_{1}(Y 40=H 195)$ both achieve the greatest ISNR (resp., PSNR) values for the length of blurring satisfying $n>45$. It can be, again, observed that both ISNR and PSNR values derived applying $E_{1}(Y 0)$ behave in 


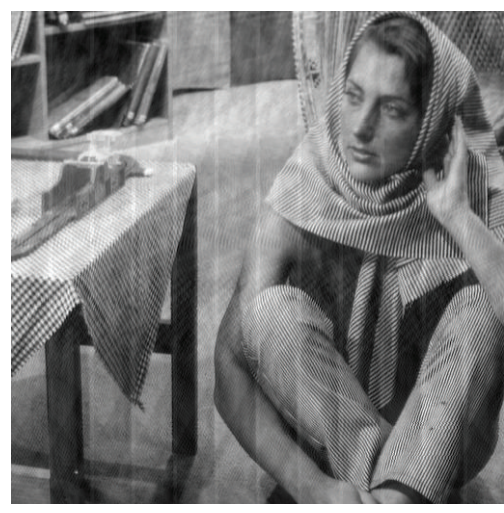

(a)

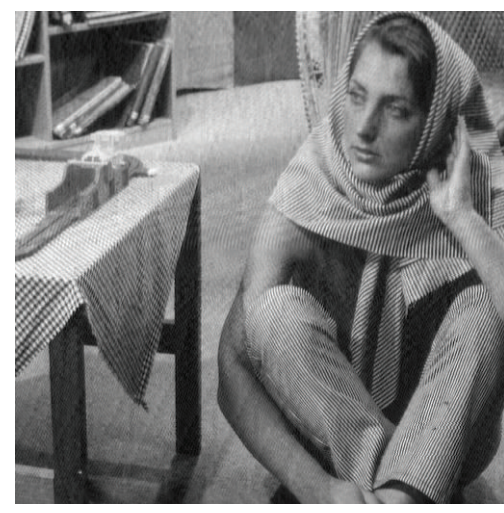

(b)

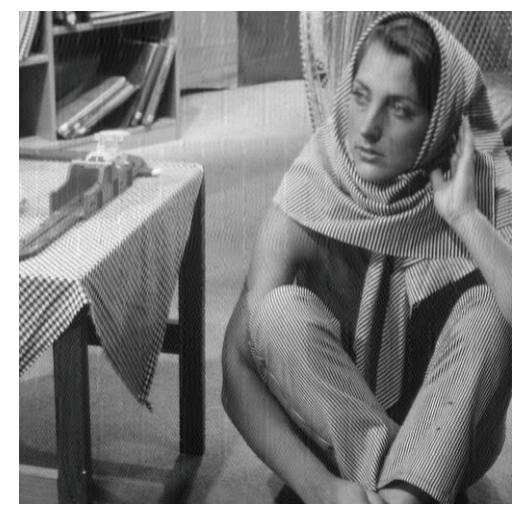

(c)

Figure 9: Restoration of Barbara for (a) Moore-Penrose, (b) Haar basis $(\kappa=40)$, and (c) Fourier basis $(\kappa=40)$.

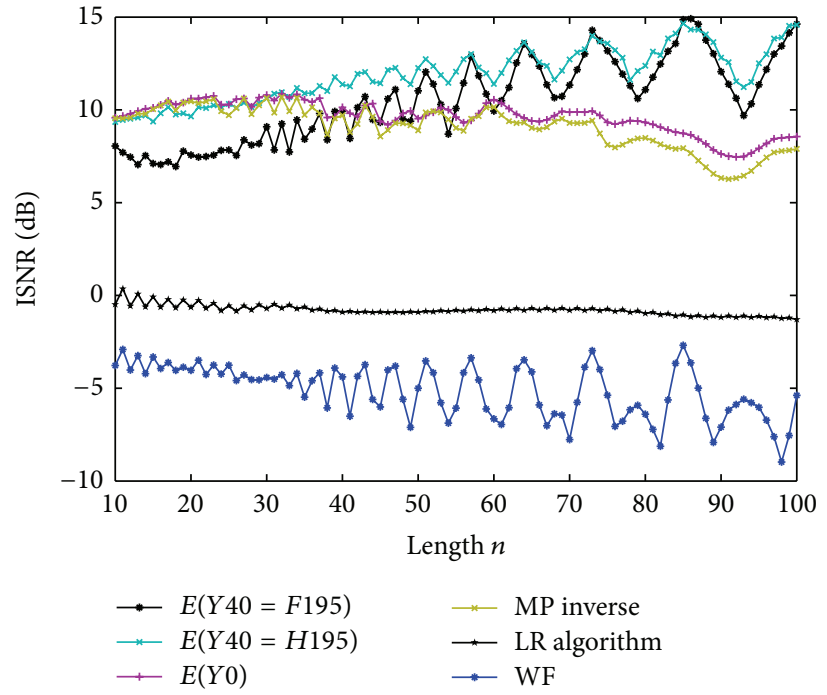

(a)

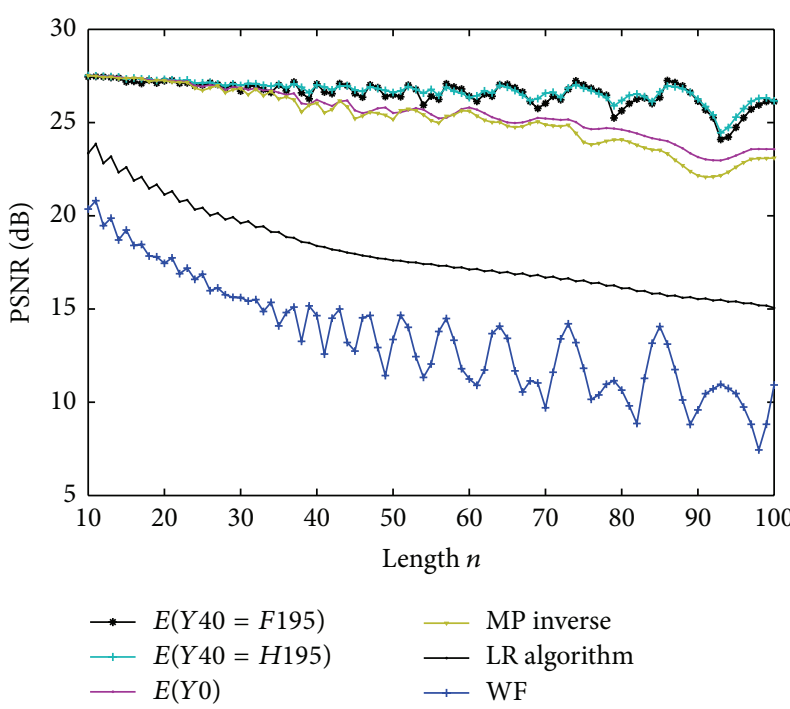

(b)

FIGURE 10: Lena image: (a) maximal ISNR versus length of blurring after 40 steps of Algorithm 6; (b) maximal PSNR versus length of blurring after 40 steps of Algorithm 6.

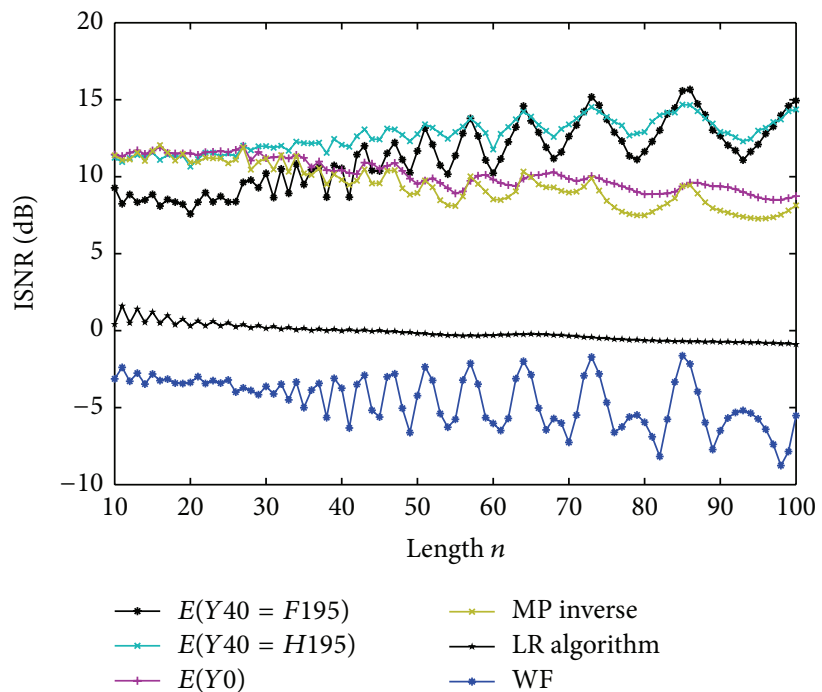

(a)

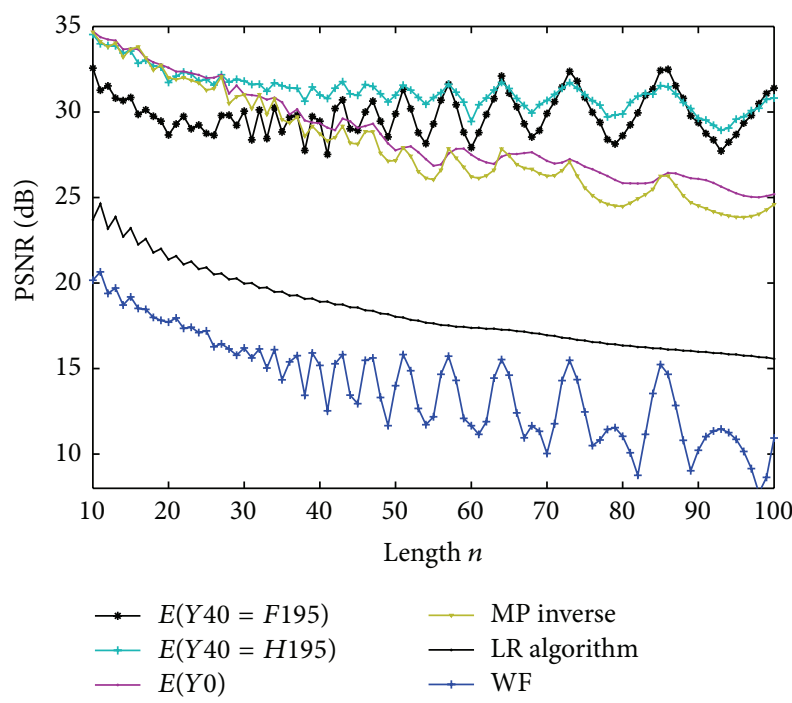

(b)

FIGURE 11: Cameraman image: (a) maximal ISNR versus length of blurring after 40 steps of Algorithm 6; (b) maximal PSNR versus length of blurring after 40 steps of Algorithm 6. 


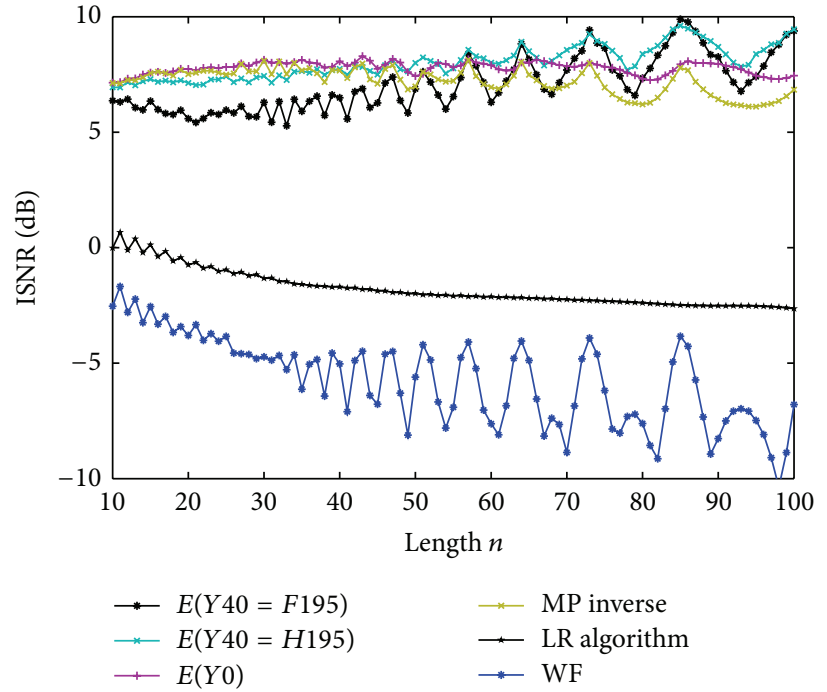

(a)

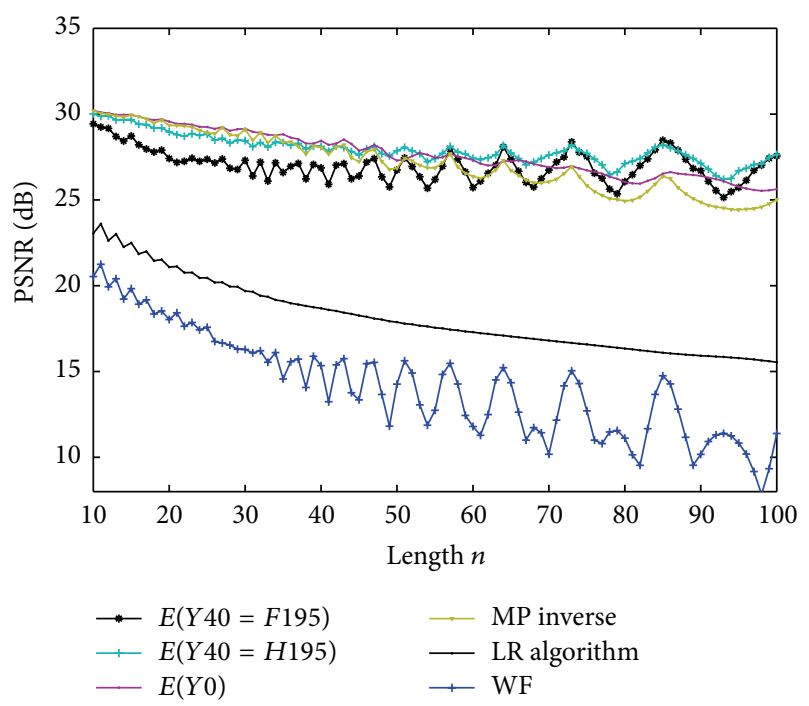

(b)

FIGURE 12: Boat image: (a) maximal ISNR versus length of blurring after 40 steps of Algorithm 6; (b) maximal PSNR versus length of blurring after 40 steps of Algorithm 6.

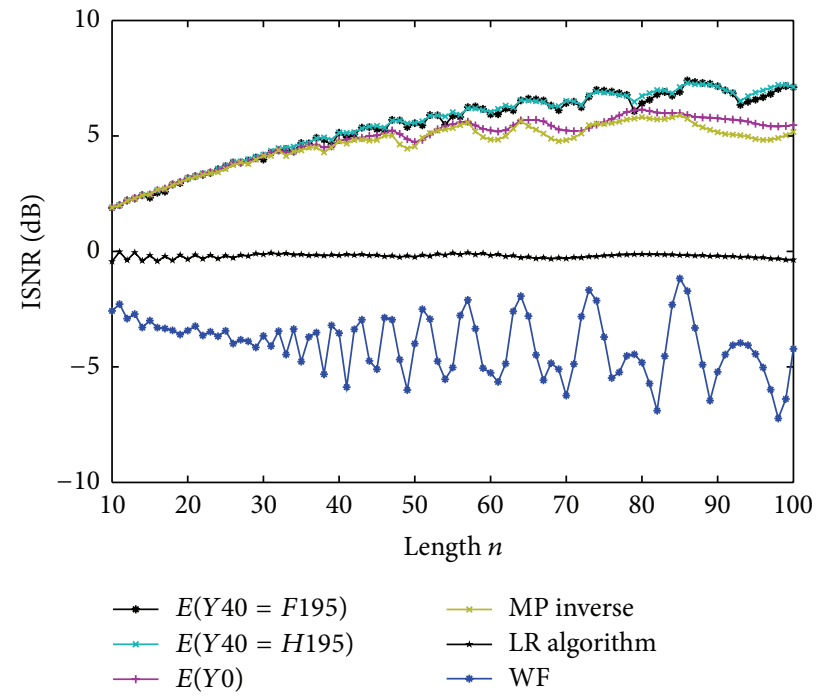

(a)

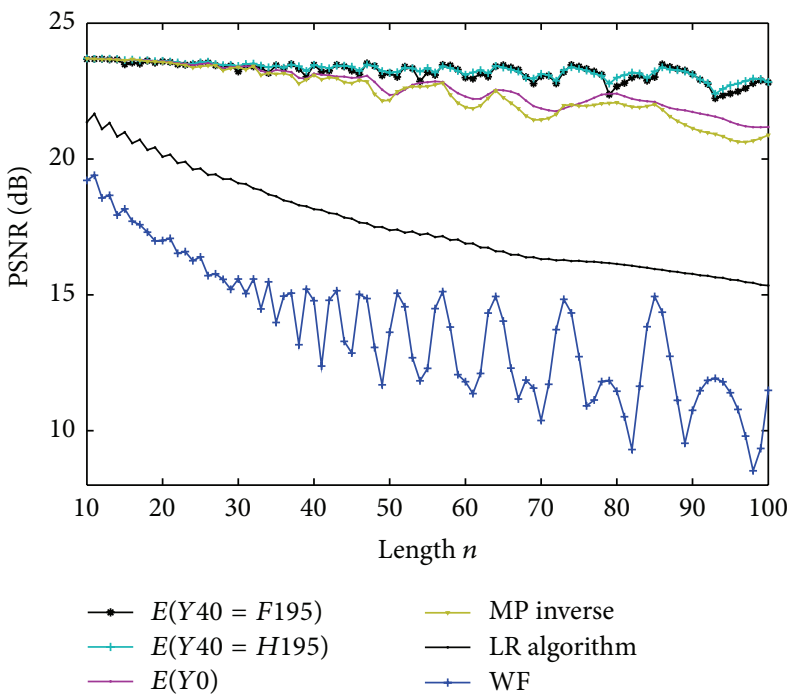

(b)

FIGURE 13: Barbara image: (a) maximal ISNR versus length of blurring after 40 steps of Algorithm 6; (b) maximal PSNR versus length of blurring after 40 steps of Algorithm 6.

a similar way as the corresponding restorations generated by the Moore-Penrose inverse solution. Clearly, WF and LR algorithms show the worst performances in symbiosis with Algorithm 6.

Confirmation of our results is presented in Figures 11 and 12 for the "salt and paper" noise of density 0.03 , as well as in Figures 13 and 14 for the Gaussian white noise of mean 0 and the variance 0.01 .

Example 12. The tested image Lena with added "salt and paper" noise with noise density of 0.03 is shown in Figure 15(a). The image that has been additionally degraded by a uniform horizontal linear motion of length 73 is presented in Figure 15(b).

Figure 16(a) illustrates restoration based on the Wiener filter, Figure 16(b) illustrates restoration generated by the Lucy-Richardson algorithm, and Figure 16(c) is the restoration defined by the Moore-Penrose solution (4).

The differences in Figures 16(a) and 16(b) as opposed to Figure 16(c) are visible by a human eye. In addition, the Moore-Penrose inverse approach achieves a better restoration than the WF and LR algorithms.

Figures 17(a) and 17(b) present restorations derived applying $\kappa=40$ times the least squares operator $E_{1}$ in conjunction 


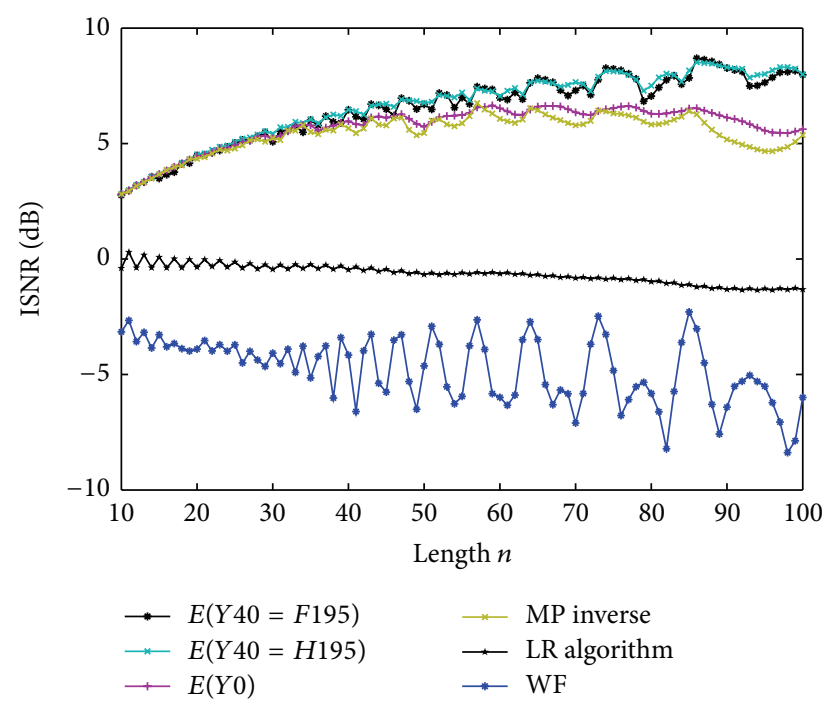

(a)

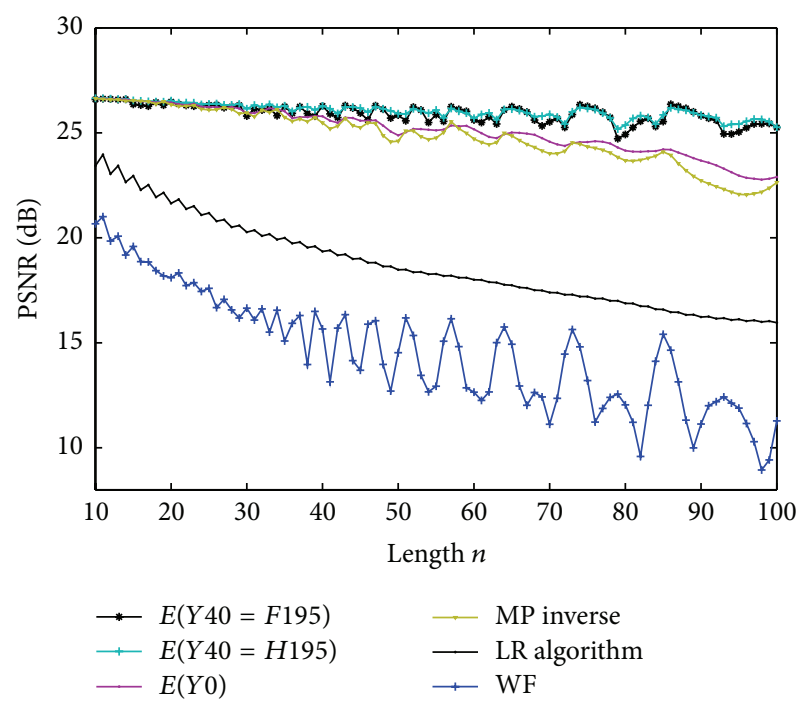

(b)

FIGURE 14: Man image: (a) maximal ISNR versus length of blurring after 40 steps of Algorithm 6; (b) maximal PSNR versus length of blurring after 40 steps of Algorithm 6.

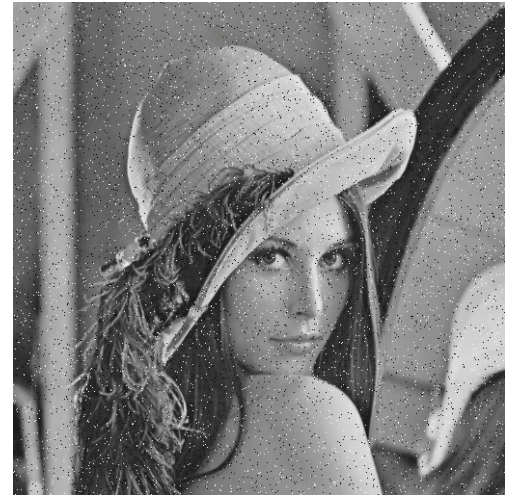

(a)

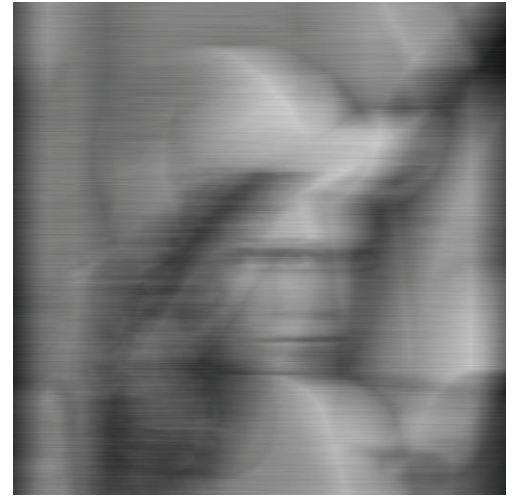

(b)

FIGURE 15: (a) Noisy image of Lena; (b) blurred noisy image of Lena.

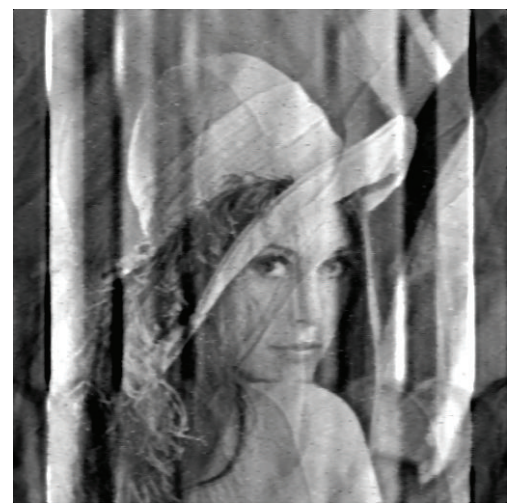

(a)

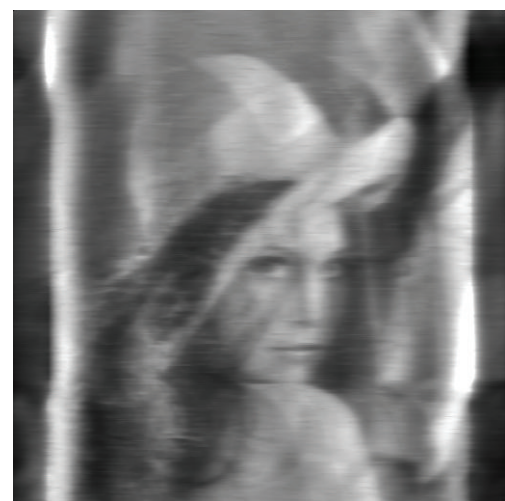

(b)

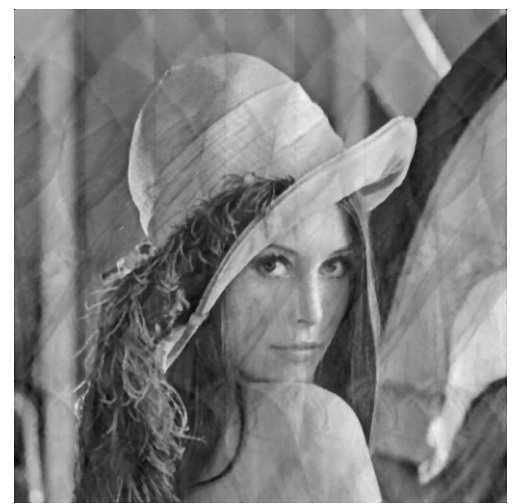

(c)

FIGURE 16: Restoration of Lena with (a) WF, (b) LR algorithm, and (c) Moore-Penrose. 


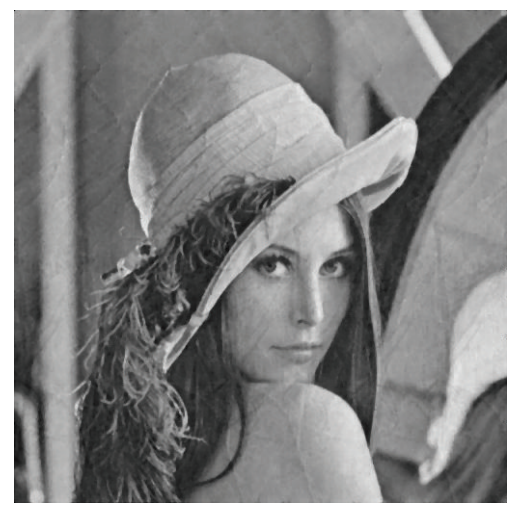

(a)

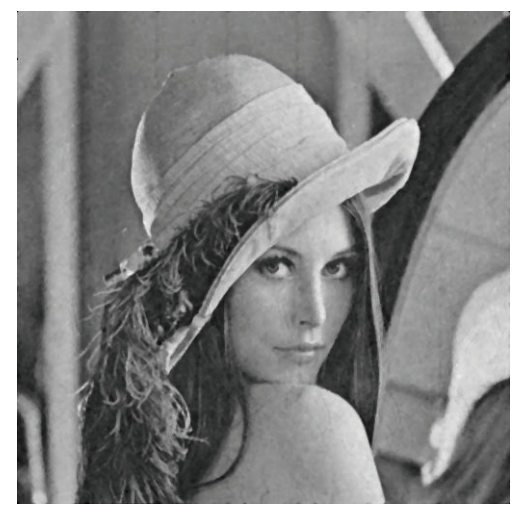

(b)

Figure 17: Restoration of Lena with (a) Haar basis and (b) Fourier basis $(\kappa=40)$.

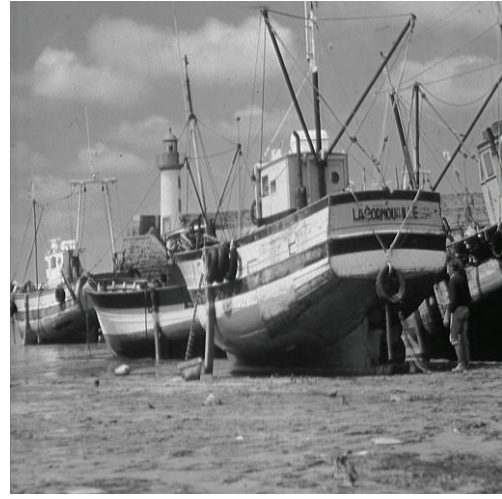

(a)

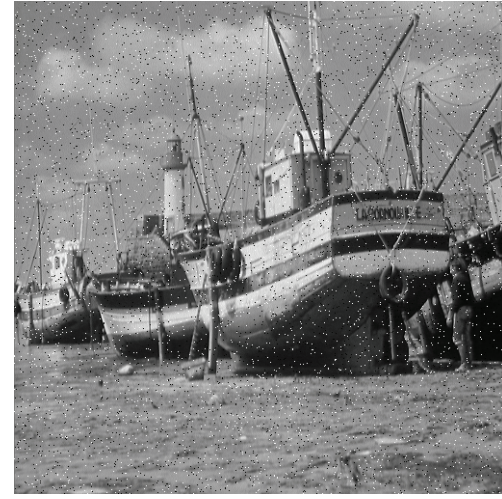

(b)

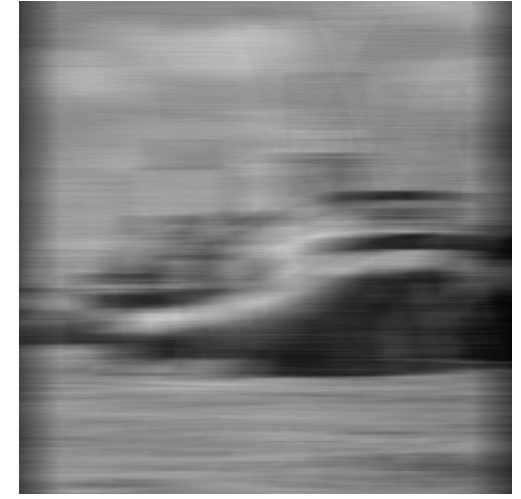

(c)

FIGURE 18: (a) Image of Boat; (b) noisy image with "salt and paper" noise with noise density of 0.03; (c) blurred noisy image degraded by a uniform horizontal linear motion of length 85 .

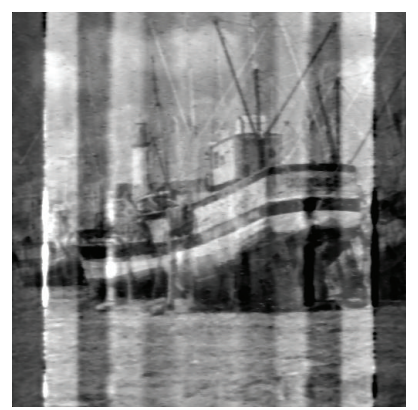

(a)

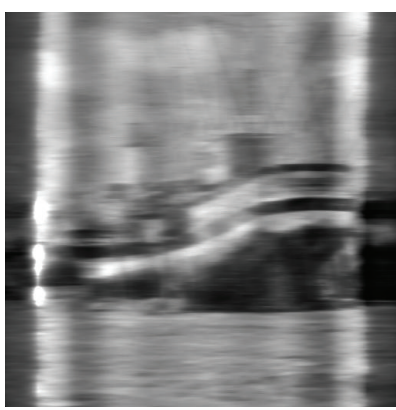

(b)

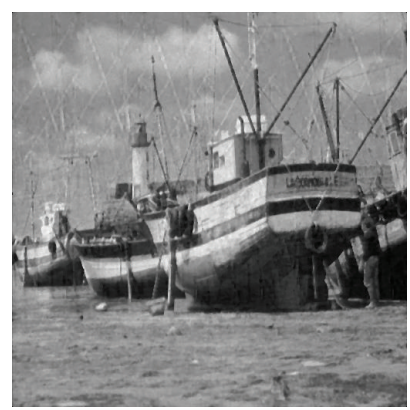

(c)

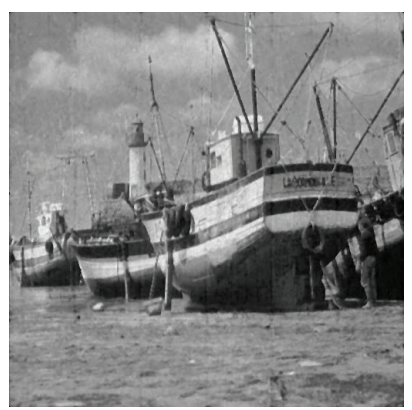

(d)

FIGURE 19: Restoration of Boat with (a) WF, (b) LR algorithm, (c) Haar basis $(\kappa=10)$, and (d) Fourier basis $(\kappa=40)$.

with the Haar and Fourier basis restoration with the basis 195, respectively. The length of blurring is again equal to 73 .

The conclusion in this example is that the better restoration is gained by using the Haar and Fourier basis (Figure 17) compared to the methods used in Figure 16 and that the improvement is observable by the human eye.

Visual confirmation of the previous conclusion is also observable from Figures 18, 19, 20, and 21.
Example 13. In this example, the previously exploited methods are tested on images degraded by different types of noise. The tested image Lena with added Gaussian white noise of mean 0 and variance 0.01 is presented in Figure 22(a) and blurred noisy image that has been degraded by a uniform linear horizontal motion of length 86 is presented in Figure 22(b).

Accordingly, a rotationally symmetric Gaussian low-pass filter of size 3 with standard deviation 45 is used for filtering. 


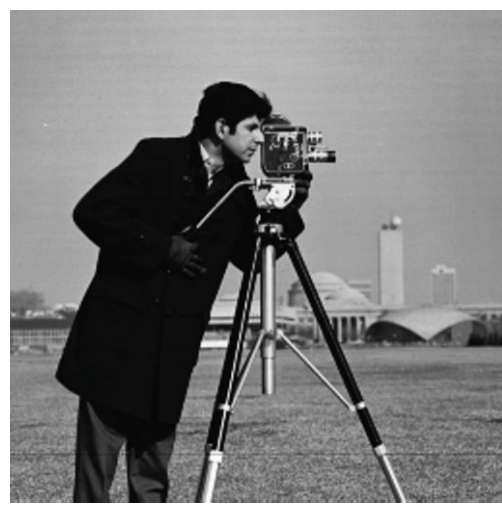

(a)

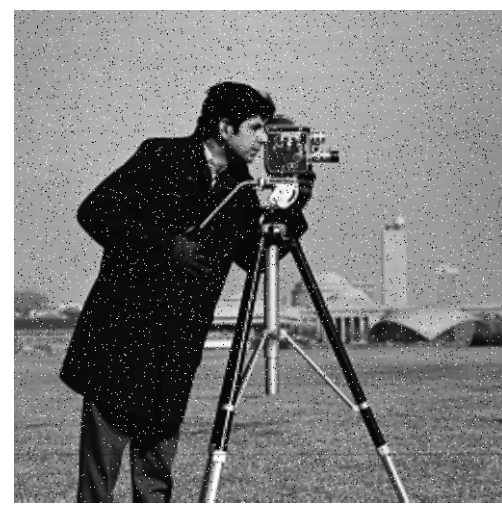

(b)

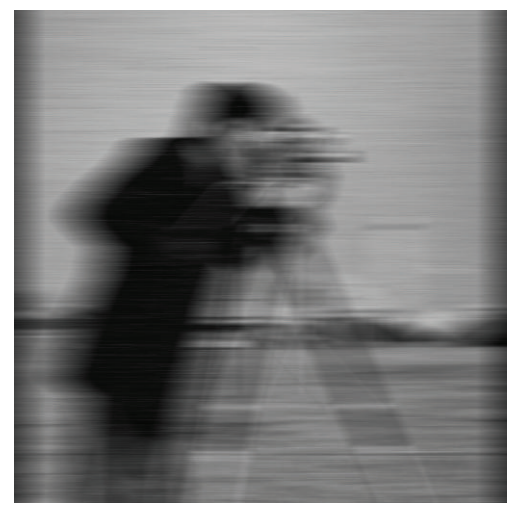

(c)

FIGURE 20: (a) Image of cameraman; (b) noisy image with "salt and paper" noise with noise density of 0.03; (c) blurred noisy image degraded by a uniform horizontal linear motion of length 64 .

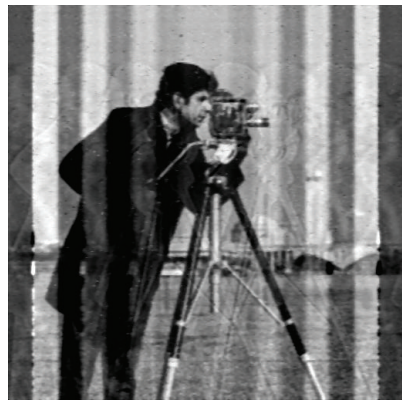

(a)

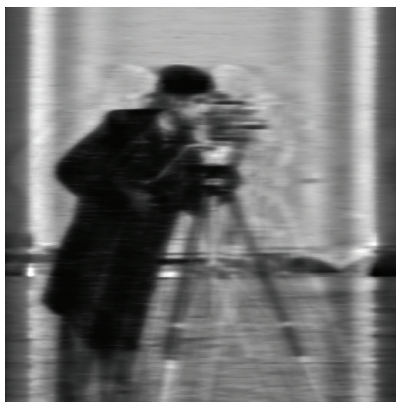

(b)

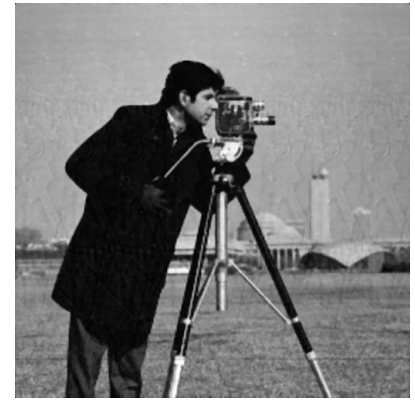

(c)

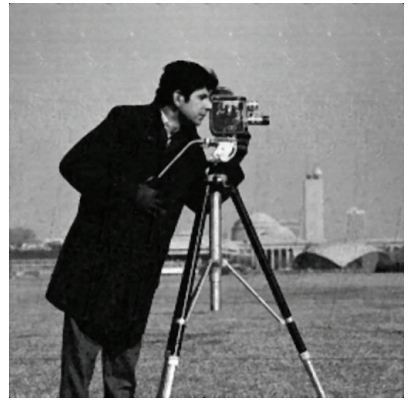

(d)

FIGURE 21: Restoration of Cameraman with (a) WF, (b) LR algorithm, (c) Haar basis $(\kappa=10)$, and (d) Fourier basis $(\kappa=40)$.

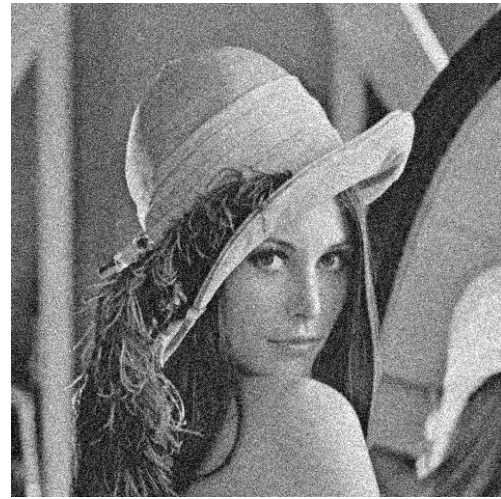

(a)

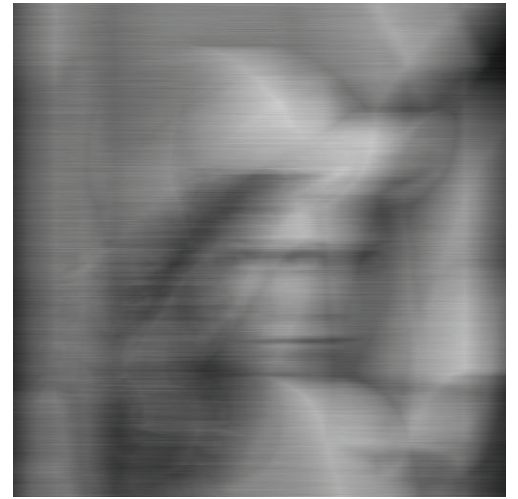

(b)

FIgURE 22: (a) Noisy image of Lena; (b) blurred and noisy image of Lena.

Restorations of Figure 22(b) based on the Wiener filter are illustrated in Figure 23(a). Figure 23(b) illustrates the restoration based on the Lucy-Richardson algorithm and Figure 23(c) illustrates the restoration based on the MoorePenrose inverse.

Restoration of Figure 22(b) based on $\kappa=40$ applications of the least squares solution $E_{1}$ in symbiosis with the Haar and Fourier basis is presented in Figures 24(a) and 24(b), respectively. It is observable that these restorations are more efficient than the restorations illustrated in Figure 23.

Confirmation of the previous conclusion (corresponding to Lena image) is also valid for Figures 25, 26, 27, and 28.

3.3. Application of the Method on Gaussian Blurred and Noisy Images. We also paid consideration to Gaussian blurring function, because it models the blurring that is caused by 


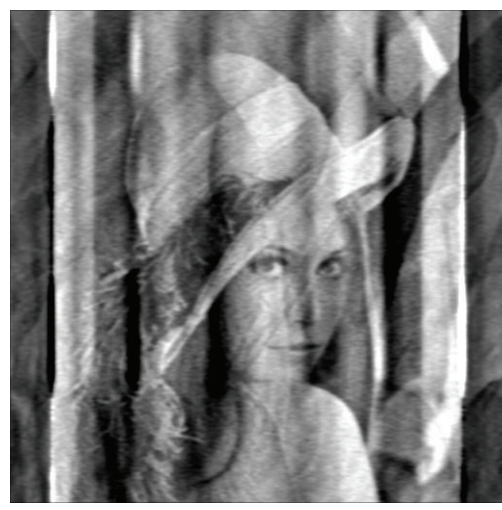

(a)

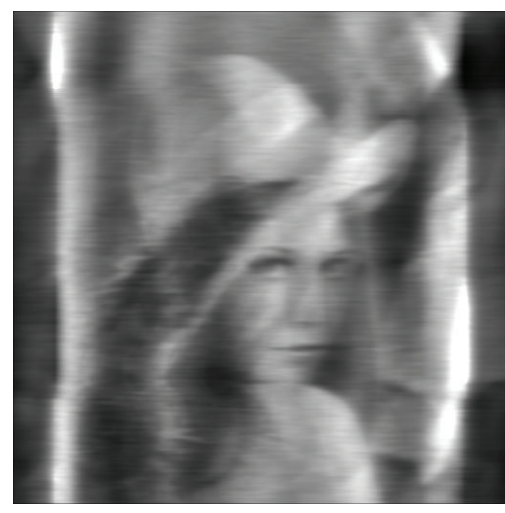

(b)

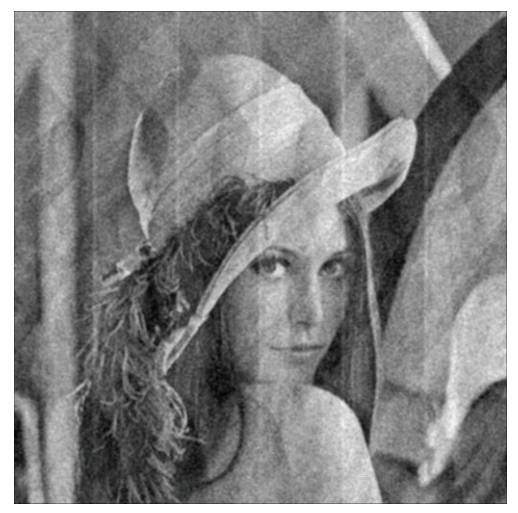

(c)

FIgURE 23: Restoration of Lena with (a) WF, (b) LR algorithm, and (c) Moore-Penrose.

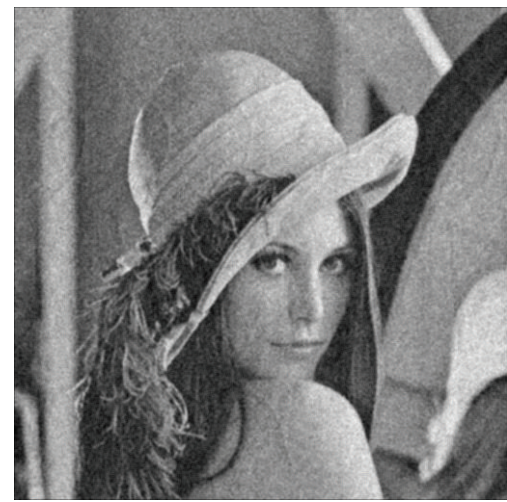

(a)

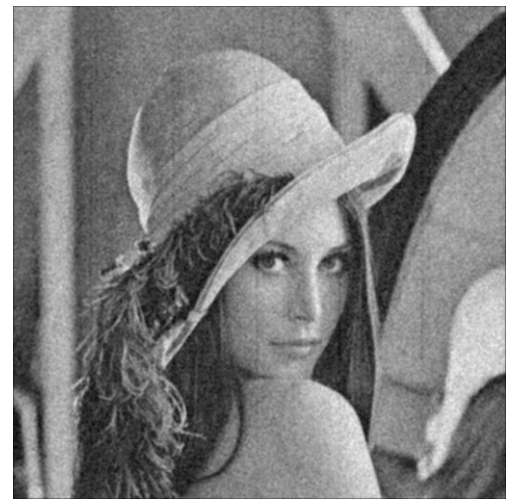

(b)

FIGURE 24: Restoration of Lena with (a) Haar basis and (b) Fourier basis.

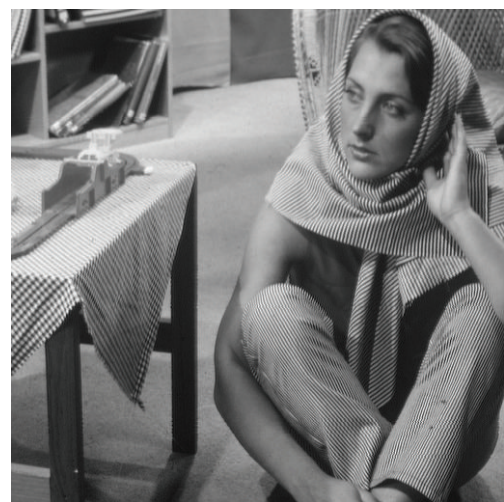

(a)

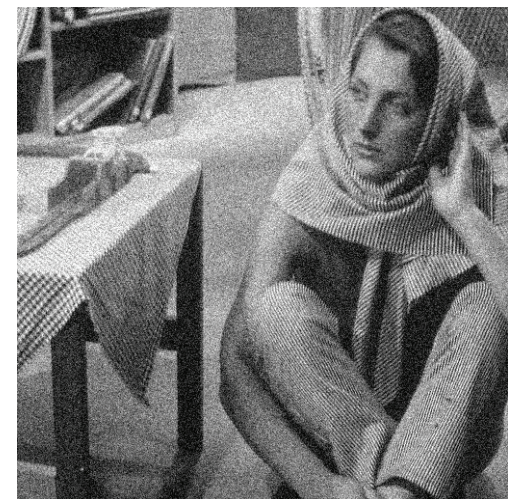

(b)

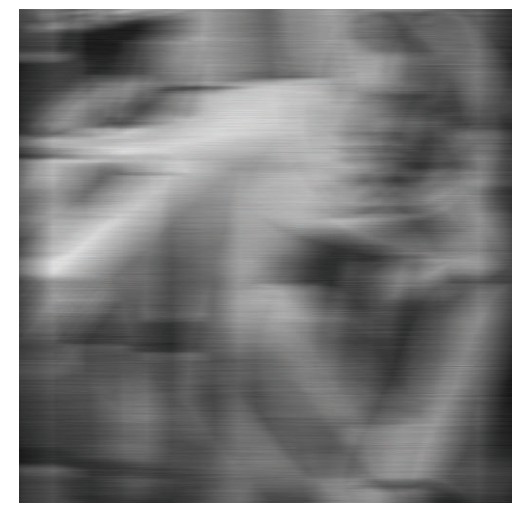

(c)

Figure 25: (a) Image of Barbara; (b) noisy image with Gaussian white noise of mean 0 and variance 0.01; (c) blurred noisy image degraded by a uniform horizontal linear motion of length 70 .

atmospheric turbulence, out-of-focus, and motion of the camera [22]. The vector $h=\left[h_{1}, h_{2}, \ldots, h_{l}\right]$ in Gaussian blur model is equal to

$$
h=[\gamma(-p), \ldots, \gamma(0), \ldots, \gamma(k)],
$$

where $\gamma(i)=e^{-i^{2} /\left(2 s^{2}\right)}, p=\lfloor n / 2\rfloor, k=\lceil n / 2\rceil$, and the parameter $s$ represents the width of the blurring function.

Example 14. In this example, we illustrate the results for different images when the Gaussian blur is present. The ISNR 


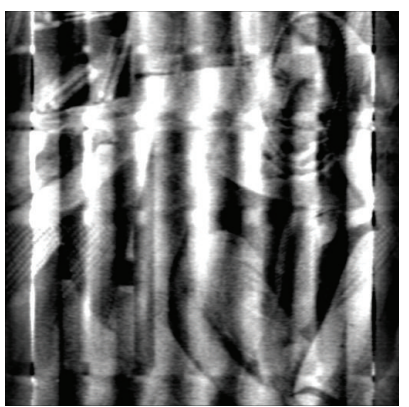

(a)

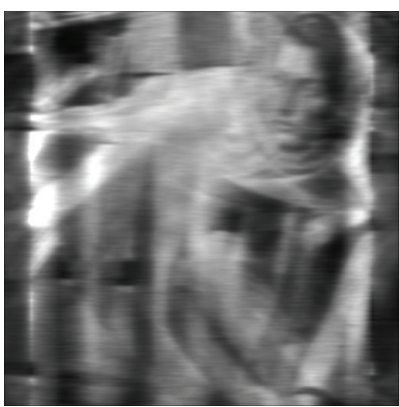

(b)

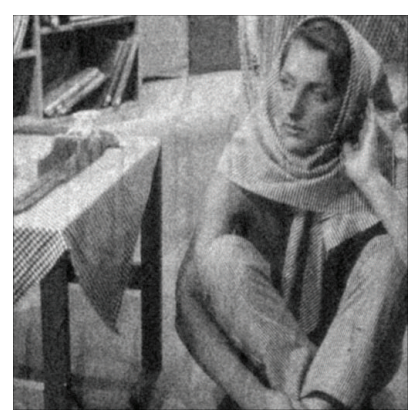

(c)

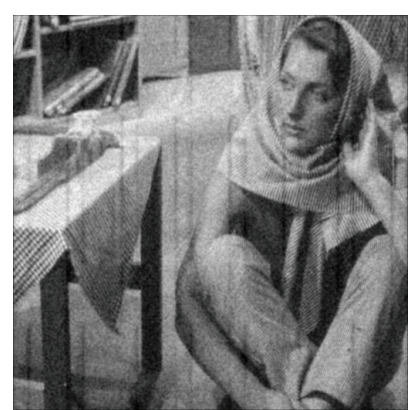

(d)

Figure 26: Restoration of Barbara with (a) WF, (b) LR algorithm, (c) Haar basis ( $\kappa=10$ ), and (d) Fourier basis $(\kappa=40)$.

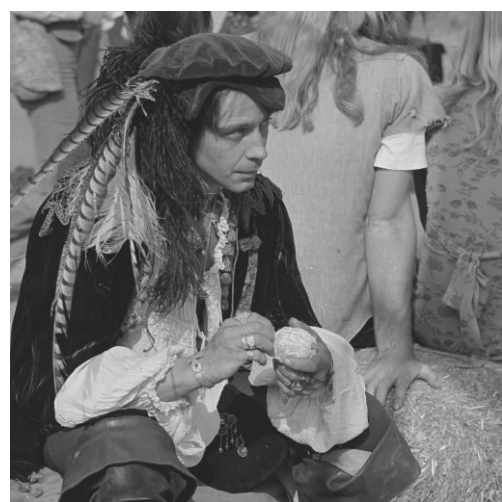

(a)

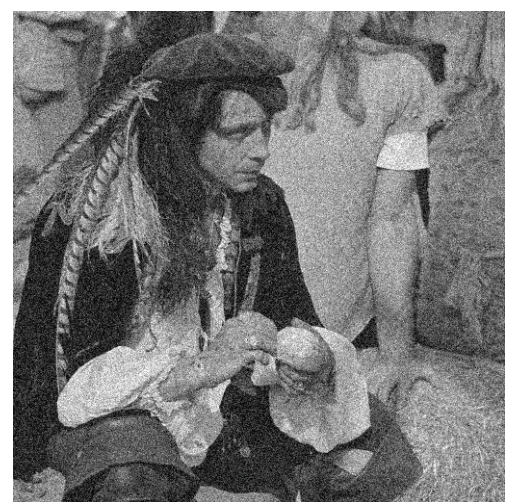

(b)

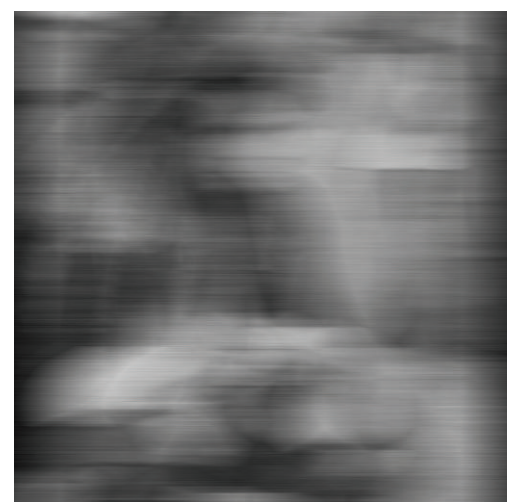

(c)

Figure 27: (a) Image of Barbara; (b) noisy image with Gaussian white noise of mean 0 and variance 0.01; (c) blurred noisy image degraded by a uniform horizontal linear motion of length 95 .

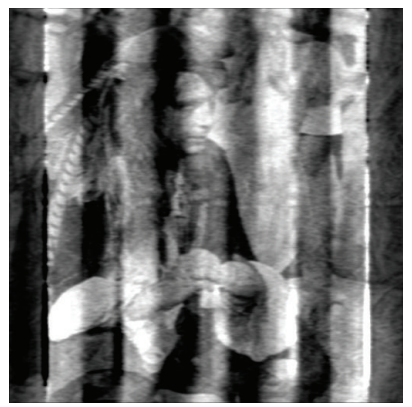

(a)

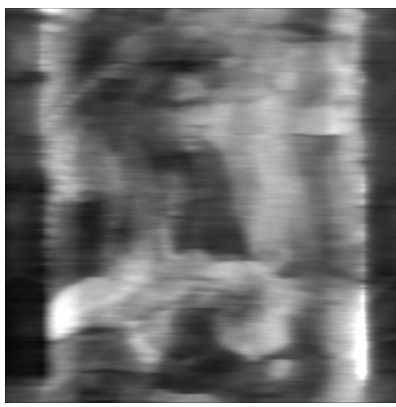

(b)

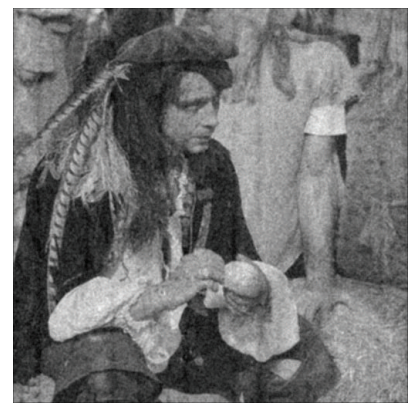

(c)

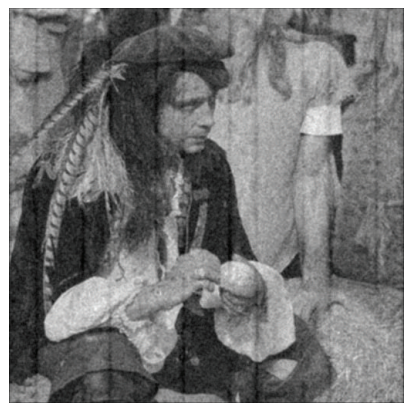

(d)

FIgURE 28: Restoration of Barbara with (a) WF, (b) LR algorithm, (c) Haar basis $(\kappa=10)$, and (d) Fourier basis $(\kappa=40)$.

and PSNR values in case of Gaussian blur are shown in Figures 29 and 30.

Figures 31 and 32 show the results for the Lena image.

The results for the Man image are shown on Figures 33 and 34 .

Example 15. The results of ISNR and PSNR in the case of Gaussian blur and Gaussian noise are presented in Figures 35 and 36 .

\section{Conclusion}

In $[9,10]$, it was shown that the application of the least squares solutions of matrix equations is a very useful tool in the image deblurring process. Our tendency in the present paper is to ensure an iterative application of these least squares solutions. The presented numerical examples and figures illustrate that both the ISNR and PSNR values increase using successive applications of the proposed operator $E_{1}$. 


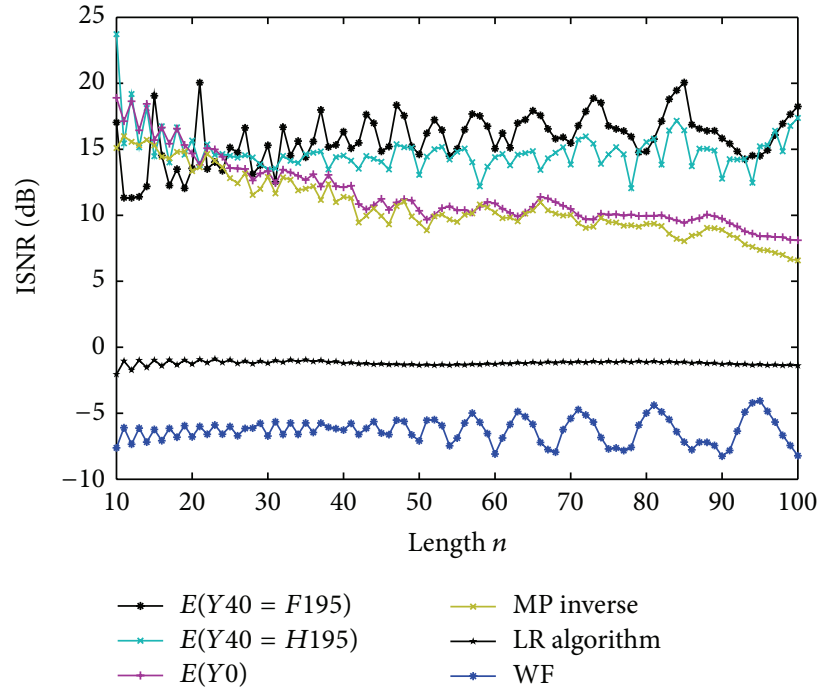

(a)

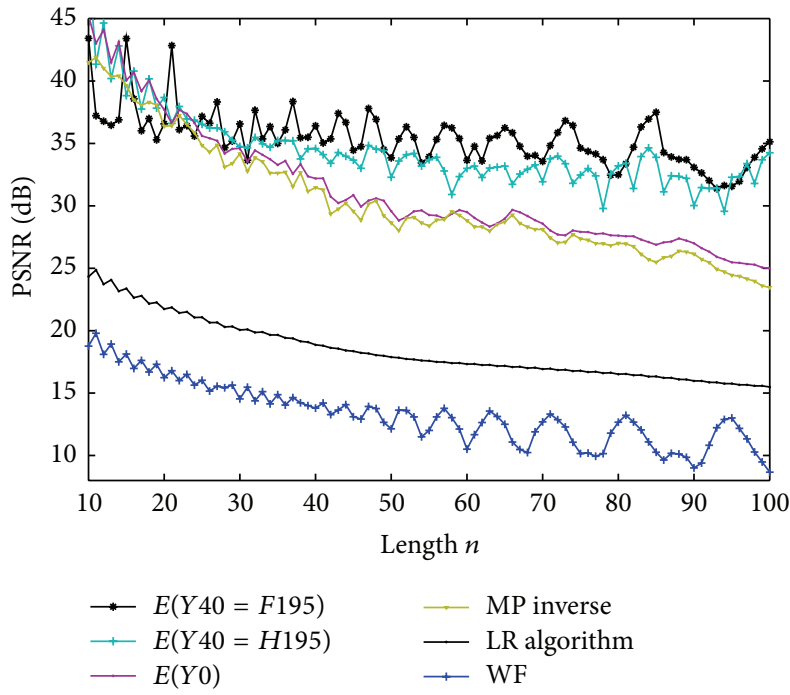

(b)

FIGURE 29: Lena image: (a) maximal ISNR versus length of blurring after 40 steps of Algorithm 1; (b) maximal PSNR versus length of blurring after 40 steps of Algorithm 1.

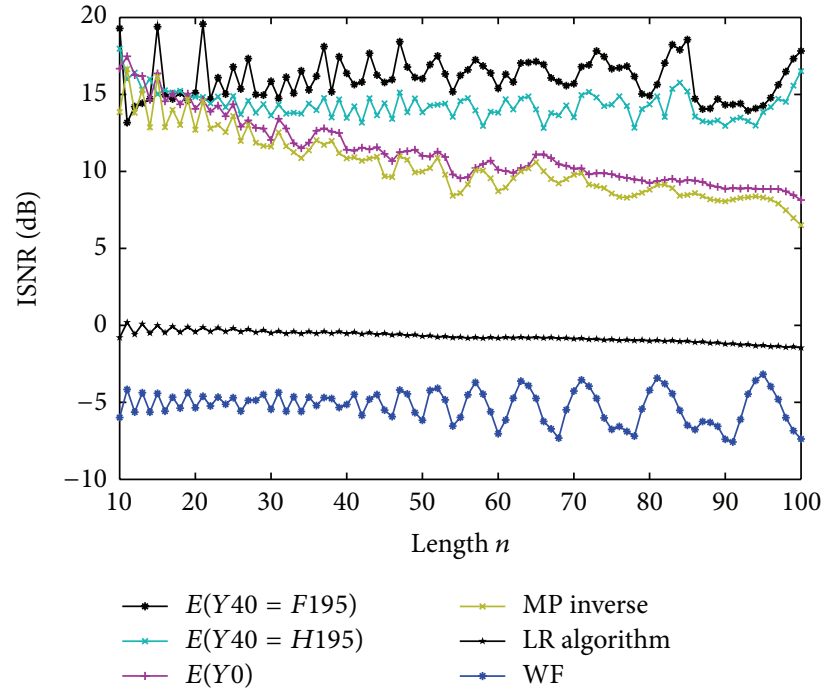

(a)

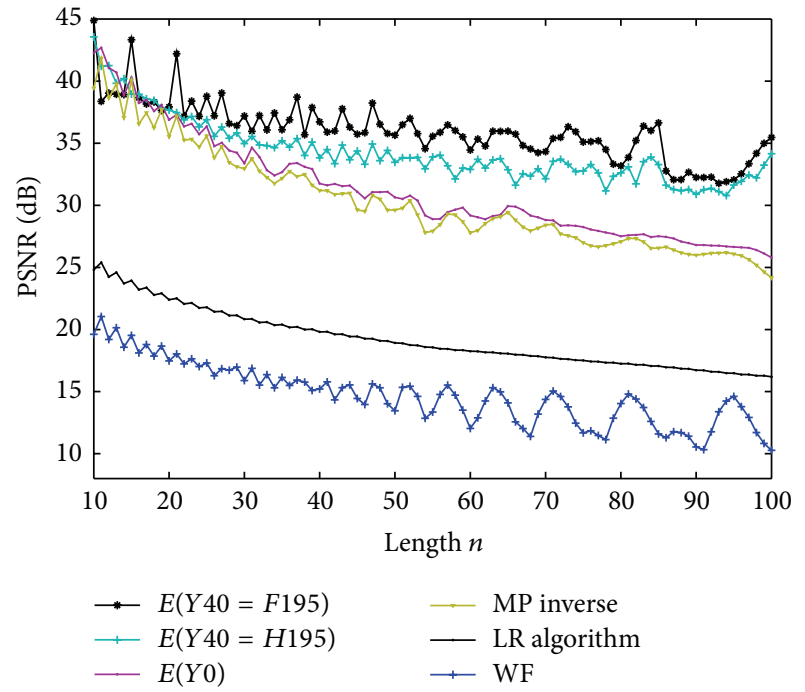

(b)

FIGURE 30: Man image: (a) maximal ISNR versus length of blurring after 40 steps of Algorithm 1; (b) maximal PSNR versus length of blurring after 40 steps of Algorithm 1.

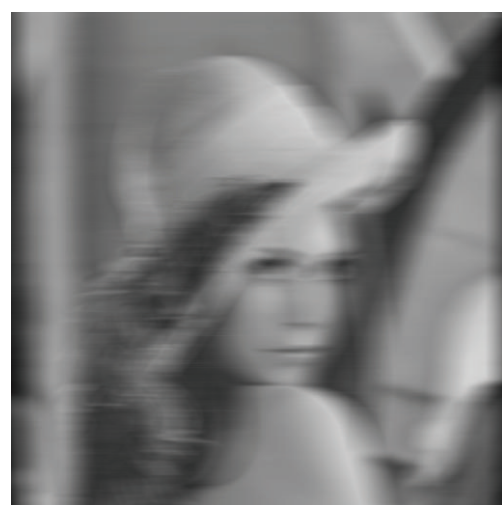

(a)

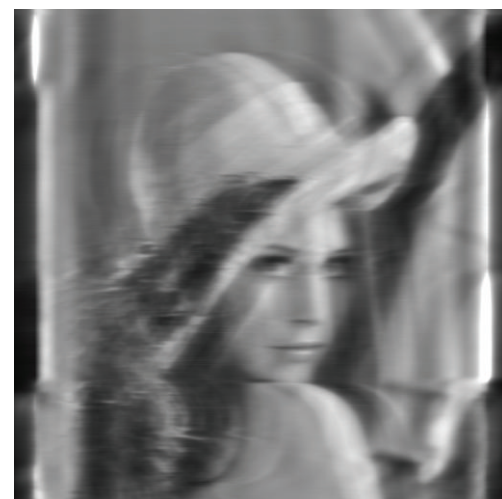

(b)

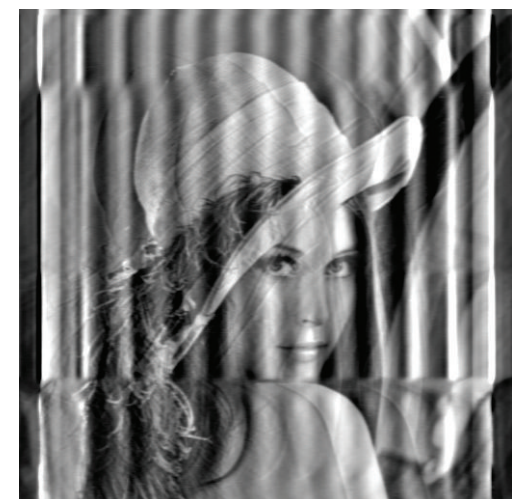

(c)

FIGURE 31: (a) Blurred image by uniform linear motion of length 42. Restoration of Lena for (b) LR algorithm and (c) WF. 


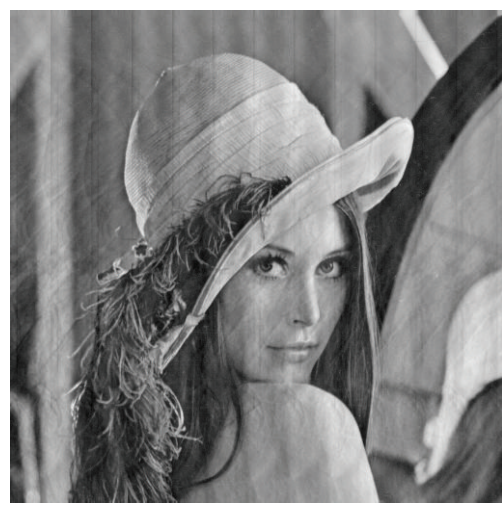

(a)

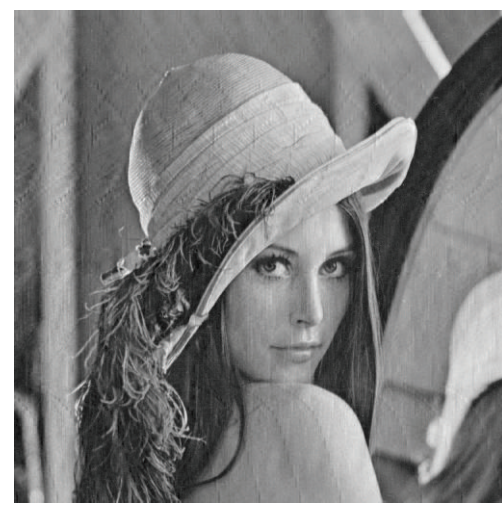

(b)

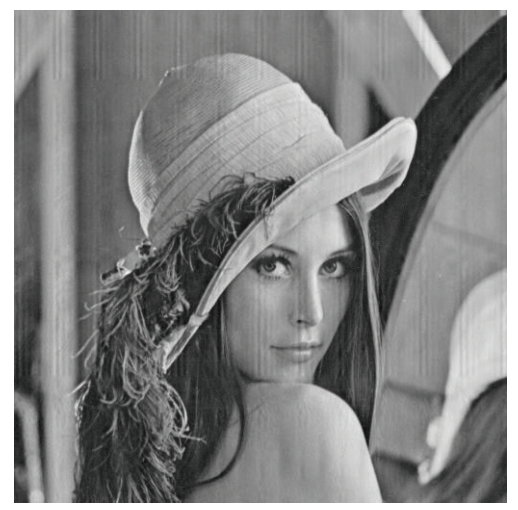

(c)

Figure 32: Restoration of Lena for (a) Moore-Penrose, (b) Haar basis $(\kappa=40)$, and (c) Fourier basis $(\kappa=40)$.

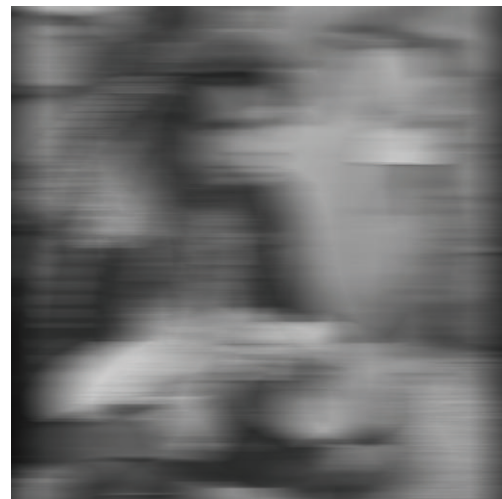

(a)

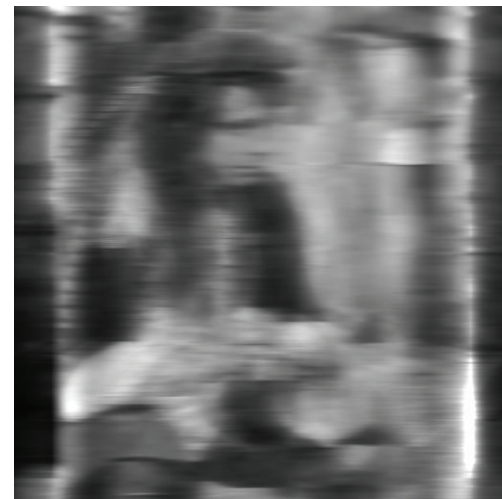

(b)

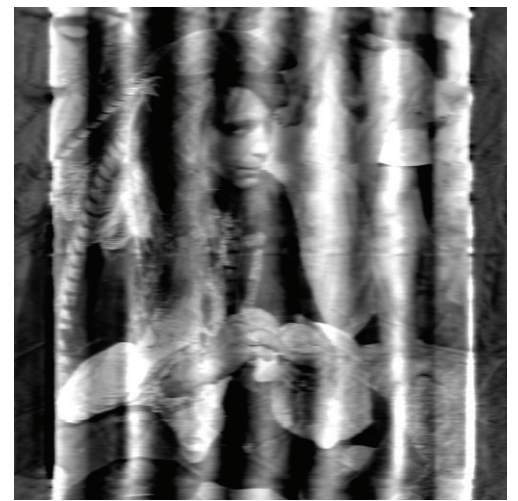

(c)

FIGURE 33: (a) Blurred image by uniform linear motion of length 76. Restoration of Man for (b) LR algorithm and (c) WF.

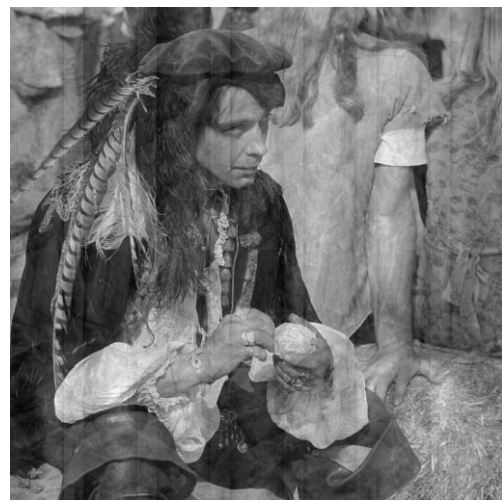

(a)

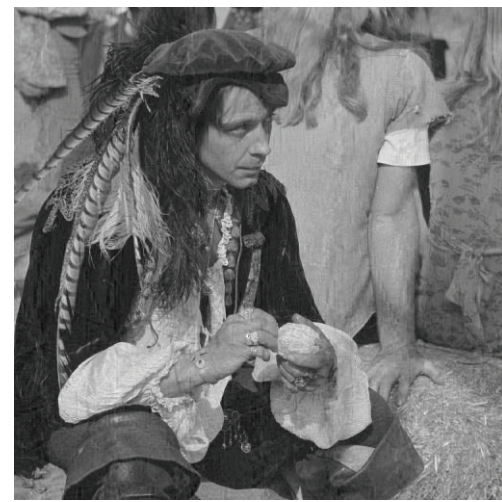

(b)

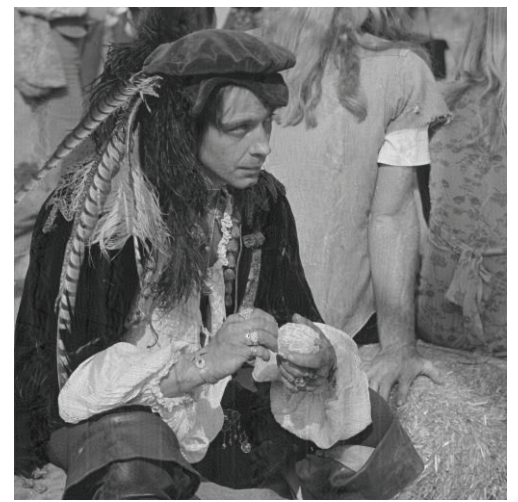

(c)

FIgURE 34: Restoration of Man for (a) Moore-Penrose, (b) Haar basis $(\kappa=40)$, and (c) Fourier basis $(\kappa=40)$.

It is clear that the proposed application of the least squares solutions of the matrix equation which models the blurring process is applicable only in symbiosis with other methods of image deconvolution. The least squares solution can be used as an improvement of other image deconvolution methods.
This symbiosis is exploited in Step (3) of Algorithm 1. Iterative application of the least squares solution $E_{1}$ on blurred and noisy images is achieved in Algorithm 6 in symbiosis with other well-known methods for image deconvolution and a selected filtering process. 


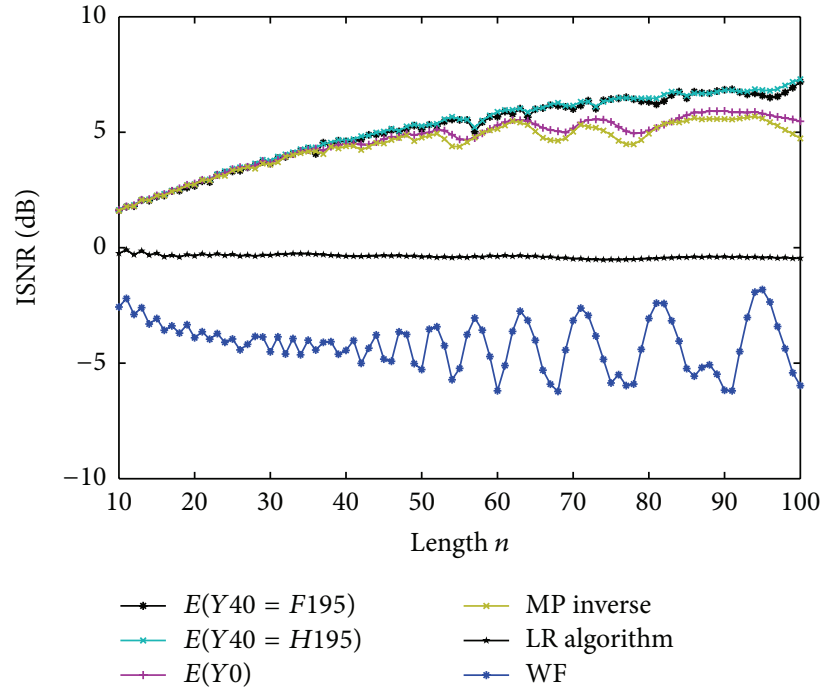

(a)

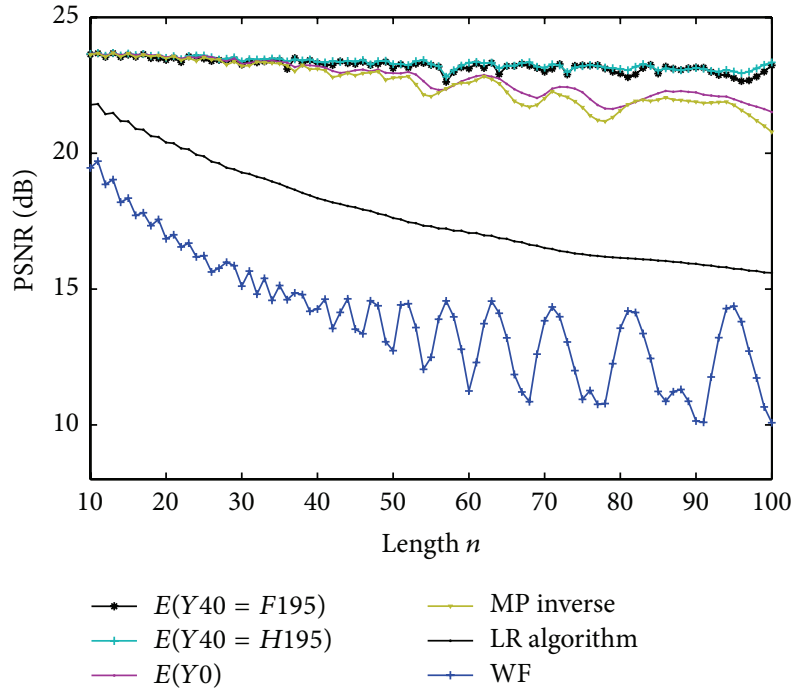

(b)

FIGURE 35: Barbara image: (a) maximal ISNR versus length of blurring after 40 steps of Algorithm 6; (b) maximal PSNR versus length of blurring after 40 steps of Algorithm 6.

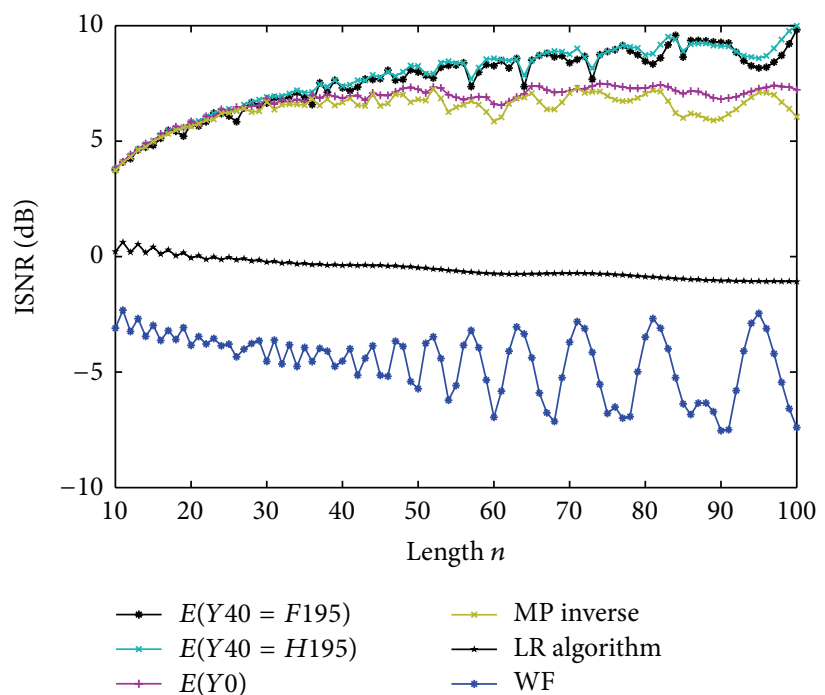

(a)

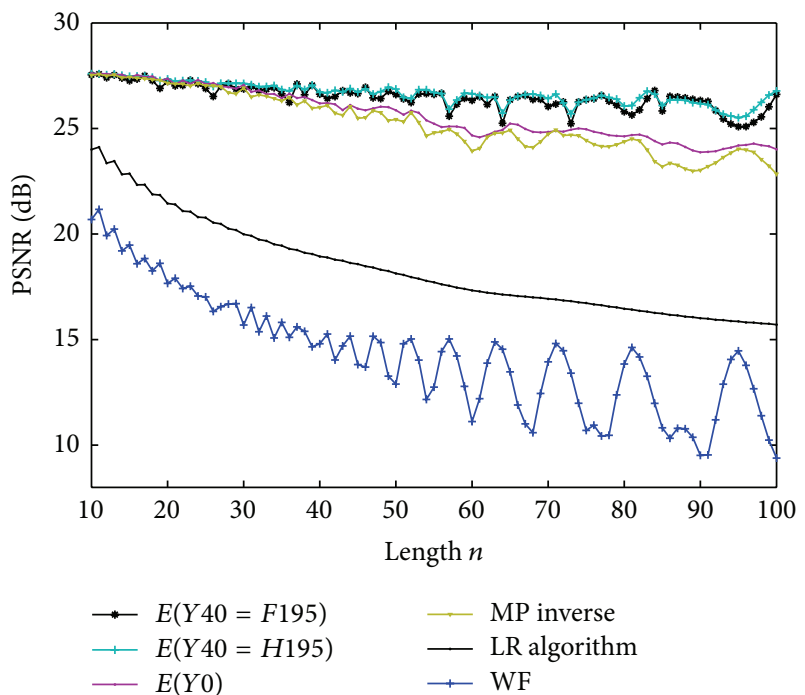

(b)

FIGURE 36: Cameraman image: (a) maximal ISNR versus length of blurring after 40 steps of Algorithm 6; (b) maximal PSNR versus length of blurring after 40 steps of Algorithm 6 .

\section{Conflict of Interests}

The authors declare that there is no conflict of interests regarding the publication of this paper.

\section{Acknowledgments}

Predrag S. Stanimirović gratefully acknowledges support from the Research Project no. 174013 of the Serbian Ministry of Science. Predrag S. Stanimirović and Igor Stojanović gratefully acknowledge support from the Project "Applying Direct Methods for Digital Image Restoring" of the Goce Delčev University.

\section{References}

[1] M. R. Banham and A. K. Katsaggelos, "Digital image restoration," IEEE Signal Processing Magazine, vol. 14, no. 2, pp. 24-41, 1997.

[2] A. Bovik, The Essential Guide to the Image Processing, Academic Press, New York, NY, USA, 2009.

[3] S. Esedoglu and S. J. Osher, "Decomposition of images by the anisotropic Rudin-Osher-Fatemi model," Communications on Pure and Applied Mathematics, vol. 57, no. 12, pp. 1609-1626, 2004.

[4] R. C. Gonzalez and R. E. Woods, Digital Image Processing, Prentice Hall, 3rd edition, 2007. 
[5] P. C. Hansen, J. G. Nagy, and D. O'Leary, Deblurring Images: Matrices, Spectra, and Filtering, SIAM, Philadelphia, Pa, USA, 2006.

[6] D. Kundur and D. Hatzinakos, "Blind image deconvolution: an algorithmic approach to practical image restoration," IEEE Signal Processing Magazine, vol. 13, no. 3, pp. 43-64, 1996.

[7] S. C. Park, M. K. Park, and M. G. Kang, "Super-resolution image reconstruction: a technical overview," IEEE Signal Processing Magazine, vol. 20, no. 3, pp. 21-36, 2003.

[8] J. van de Weijer and R. van den Boomgaard, "Least squares and robust estimation of local image structure," International Journal of Computer Vision, vol. 64, no. 2-3, pp. 143-155, 2005.

[9] P. S. Stanimirović, S. Chountasis, D. Pappas, and I. Stojanović, "Removal of blur in images based on least squares solutions," Mathematical Methods in the Applied Sciences, vol. 36, no. 17, pp. 2280-2296, 2013.

[10] P. Stanimirović, I. Stojanović, S. Chountasis, and D. Pappas, "Image deblurring process based on separable restoration methods," Computational and Applied Mathematics, vol. 33, no. 2, pp. 301-323, 2014.

[11] S. Chountasis, V. N. Katsikis, and D. Pappas, "Applications of the Moore-Penrose inverse in digital image restoration," Mathematical Problems in Engineering, vol. 2009, Article ID 170724, 12 pages, 2009.

[12] S. Chountasis, V. N. Katsikis, and D. Pappas, "Digital image reconstruction in the spectral domain utilizing the MoorePenrose inverse," Mathematical Problems in Engineering, vol. 2010, Article ID 750352, 14 pages, 2010.

[13] S. Chountasis, V. N. Katsikis, and D. Pappas, "Image reconstruction methods for MATLAB users-a Moore-Penrose inverse approach," in MATLAB-A Fundamental Tool for Scientific Computing and Engineering Applications: Volume 1, InTech, 2012.

[14] P. J. Maher, "Some operator inequalities concerning generalized inverses," Illinois Journal of Mathematics, vol. 34, no. 3, pp. 503$514,1990$.

[15] R. Penrose, "A generalized inverse for matrices," Proceedings of the Cambridge Philosophical Society, vol. 51, pp. 406-413, 1955.

[16] B. H. Shakibaei and J. Flusser, "Image deconvolution in the moment domain," in Moments and Moment Invariants-Theory and Applications, vol. 1, pp. 111-125, GCSRL, 2014.

[17] S. A. Dudani, K. J. Breeding, and R. B. McGhee, "Aircraft identification by moment invariants," IEEE Transactions on Computers, vol. 26, no. 1, pp. 39-46, 1977.

[18] P. Milanfar, W. C. Karl, and A. S. Willsky, "A moment-based variational approach to tomographic reconstruction," IEEE Transactions on Image Processing, vol. 5, no. 3, pp. 459-470, 1996.

[19] T. B. Nguyen and B. J. Oommen, "Moment-preserving piecewise linear approximations of signals and images," IEEE Transactions on Pattern Analysis and Machine Intelligence, vol. 19, no. 1, pp. 84-91, 1997.

[20] M. R. Teague, "Image analysis via the general theory of moments," Journal of the Optical Society of America, vol. 70, no. 8, pp. 920-930, 1980.

[21] M. Sonka, V. Hlavac, and R. Boyle, Image Processing, Analysis and Machine Vision, Cengage Learning, Stamford, Conn, USA, 2014.

[22] R. E. Hufnagel and N. R. Stanley, "Modulation transfer function associated with image transmission through turbulence media," Journal of the Optical Society of America, vol. 54, no. 1, pp. 52-60, 1964. 


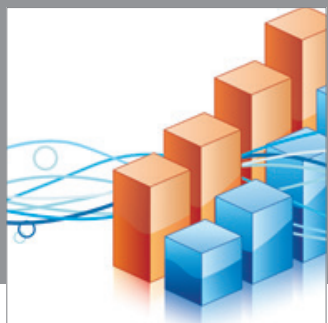

Advances in

Operations Research

mansans

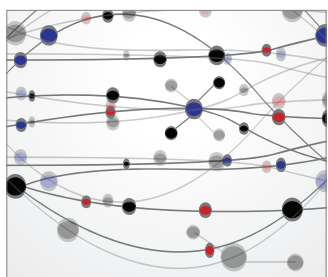

The Scientific World Journal
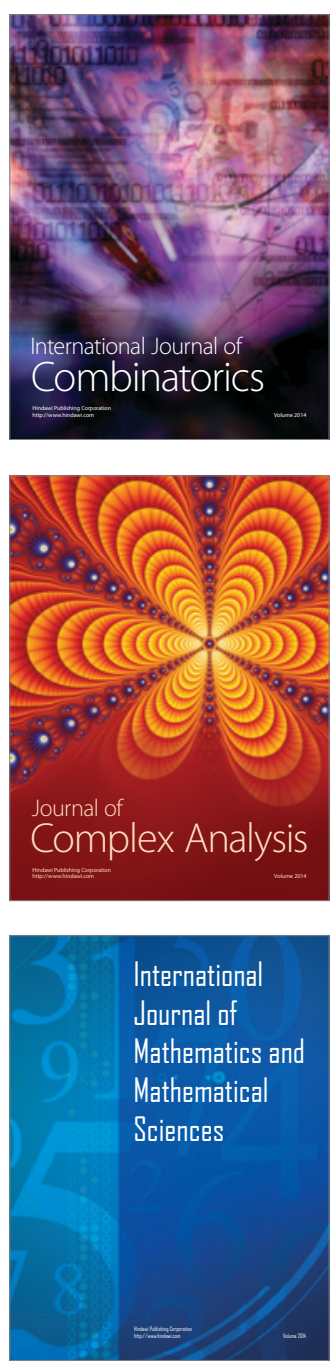
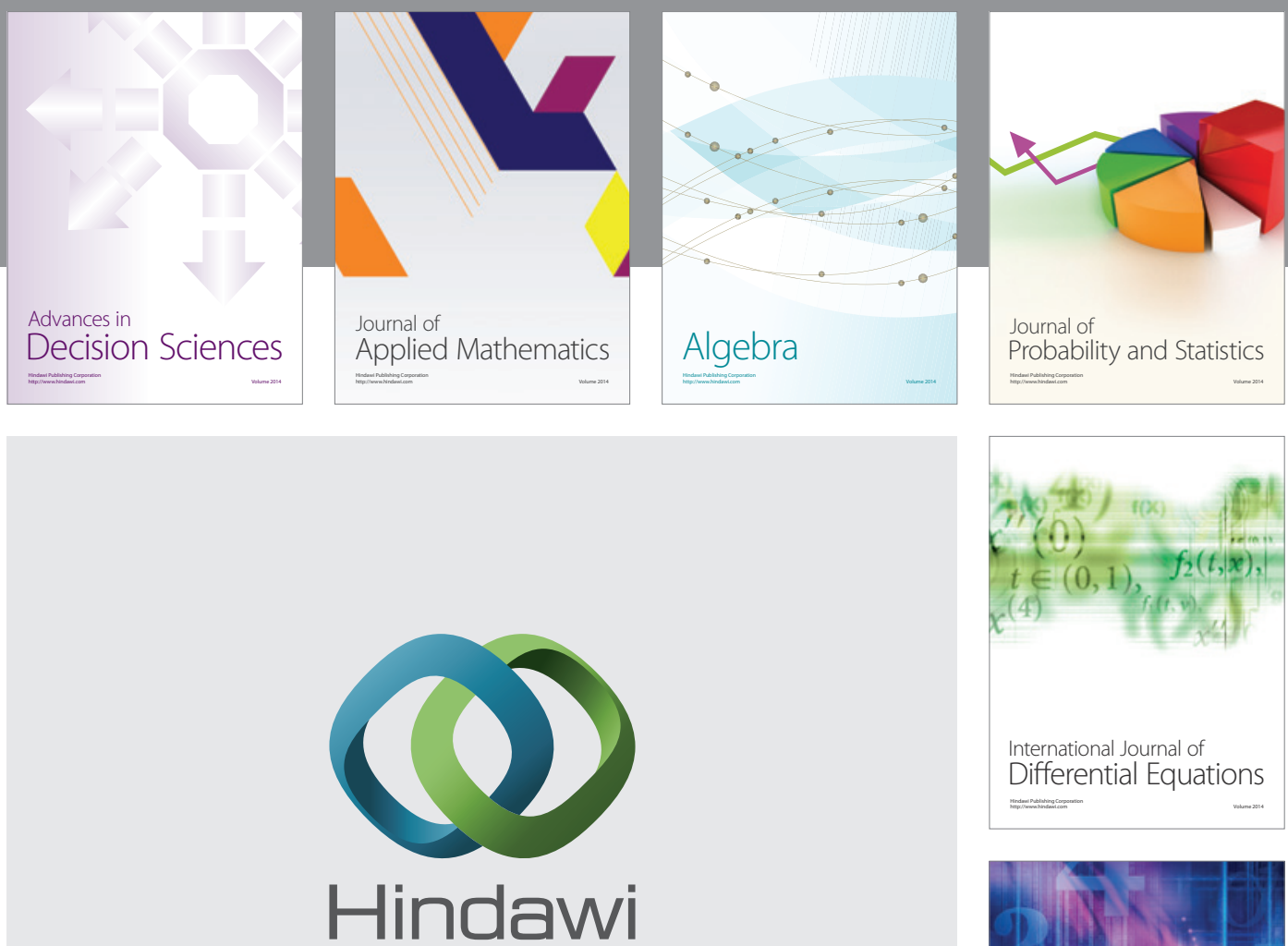

Submit your manuscripts at http://www.hindawi.com
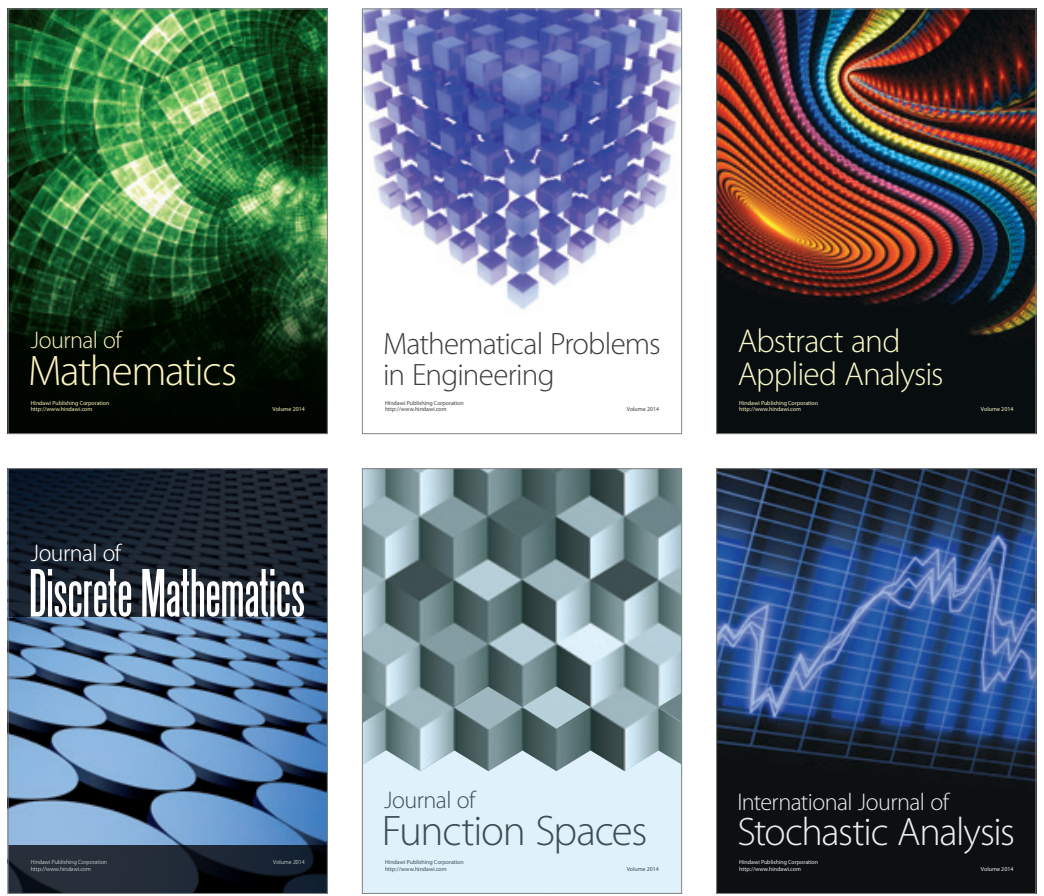

Journal of

Function Spaces

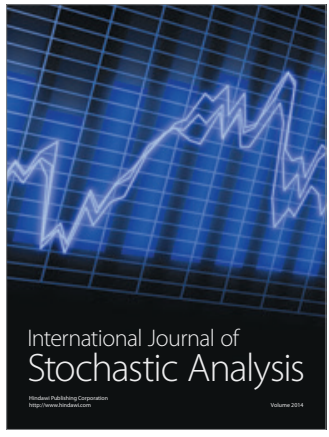

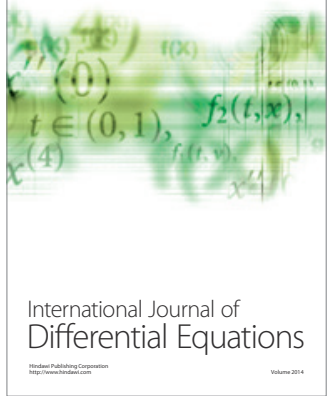
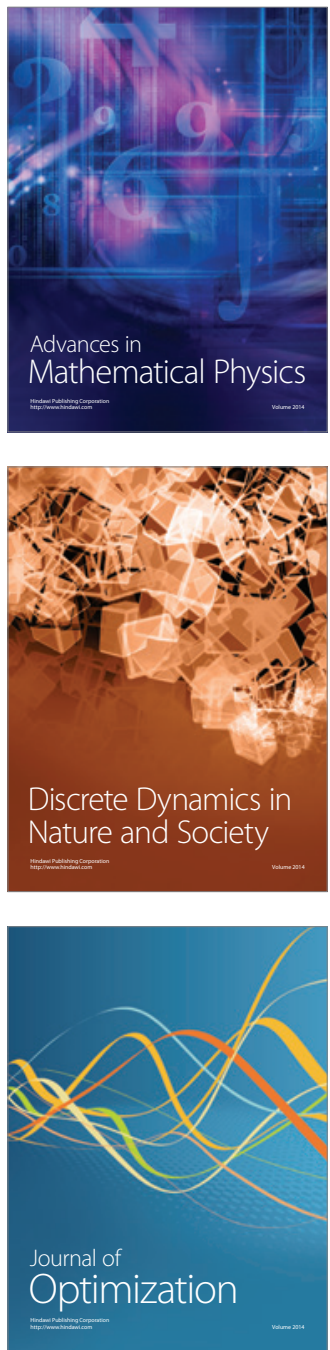